\title{
Jugend, Recht und Kriminalität
}

\section{Die soziale Bedeutung des Rechtes}

Insofern als das Recht eines der fundamentalen Instrumente darstellt, durch die Gesellschaften das grundlegende Problem der gesellschaftlichen Ordnung zu lösen versuchen, ist es nicht verwunderlich, daß Jugend und Recht und Jugend und Kriminalität in einem prekären Spannungsverhältnis stehen. Versteht man Jugend als eine besonders kritische und entscheidende Phase der lebenslangen Sozialisation, die im Idealfall in einer Einpassung des jungen Erwachsenen in die gegebene gesellschaftliche Ordnung mündet, durch die gleichzeitig kulturelle und soziale Traditionen aufrecht erhalten, aber auch neue Denk- und Verhaltensweisen eingebracht werden, die Kontinuität im Wandel oder Wandel in der Kontinuität ermöglichen, so wird das besondere Verhältnis der Jugend zum Recht verständlich. In seiner allgemeinsten Bestimmung bezeichnet Recht alle Verhaltensregeln, bei deren Übertretung eine Sanktion droht oder eine autorisierte Instanz einschreiten kann. Da in ausdifferenzierten Gesellschaften die Zahl und die Komplexität dieser Verhaltensregeln immer größer und weniger durchschaubar wird, kann man mit Fug und Recht davon ausgehen, daß der Sozialisationsprozeß vor zunehmende Schwierigkeiten gestellt wird, die geltenden Normen hinreichend transparent und verbindlich zu machen. Selbst wenn sich die Zahl jener Rechtsregeln, die Strafrechtstatbestände festlegen, kaum vergrößert hat, so wird dadurch der Sozialisationsprozeß nicht entlastet, besteht doch das eigentliche Problem für die Sozialisation nicht so sehr bzw. nicht allein darin, die entsprechenden Normen an sich zu vermitteln, sondern die Regeln zu implementieren, nach denen diese Regeln - z.B. situationsspezifisch - anzuwenden sind. Zweitens schließlich wird nach Max Weber das Recht neben der Religion, der politischen Herrschaft, der Ökonomie etc. als ganz wesentlicher Bereich der Sozialstruktur angesehen. Der Jugendliche bzw. der Heranwachsende hat in bezug auf alle diese Aspekte der Sozialstruktur diffizile Übergangspassagen zu bewältigen, die auf der kognitiven, emotionalen und motivationalen Ebene schwere Herausforderungen darstellen (vgl. z.B. Olbrich 1984; Thomae 1984; SeiffgeKrenke 1984). Insofern als das Recht die gesellschaftliche Ordnung symbolisiert, stellt es auch den Adressaten für Handlungen Jugendlicher dar, die sich mit der geltenden Ordnung (noch) nicht abfinden wollen oder können. Die Verstöße gegen geltendes Recht haben - so Durkheim (vgl. Durkheim 1961; Durkheim 1977 bzw. 1988 sowie kommentierend und empirisch prüfend dazu Albrecht 1981; Gephart 1990) - dabei immer zwei wichtige Funktionen: Auf der einen Seite stellen sie den Anlaß dar, das geltende Recht deutlich zu machen und die Solidarität der Konformen in einer emotional aufgeladenen 
Situation nachdrücklich empfinden zu lassen, auf der anderen Seite stellen die Normbrüche immer auch Anlässe dar, über die „Richtigkeit“ oder Legitimität des geltenden Rechts nachzudenken und Alternativen ins Auge zu fassen. Abweichendes Verhalten mag Resultat und Ausdruck sozialen Wandels sein, der bisher noch nicht so weit durchgedrungen ist, daß er zu einer ,neuen Moral" geführt hätte, aber der Verbrecher von gestern ist vielleicht der Moralist von morgen (vgl. Durkheim 1961, S. 159-161).

\section{Die Kriminalität der Jugendlichen und ihre rechtliche Behandlung}

\subsection{Die rechtliche Wertung des kriminellen Handelns von „Jugendlichen“ in der Vergangenheit}

Das Recht hat diesem besonderen Verhältnis von Jugend und Recht, insbesondere wenn es um das Strafrecht geht, historisch betrachtet über lange Zeit nur begrenzt Rechnung getragen, wobei natürlich nicht übersehen werden kann, daß ohne die Existenz einer Altersphase der Jugend, so wie wir sie seit geraumer Zeit kennen, die reale Situation eines „Jugendlichen" eine andere war als sie heute ist. Dennoch wurde seit jeher die ,Jugend" eines Täters als Grund für eine Milderung oder für ein Absehen von Bestrafung angesehen, allerdings lag die Altersgrenze auch im Mittelalter zunächst noch recht niedrig. So durften nach germanischem Recht im II. Jahrhundert keinem Jugendlichen unter 12 Jahren die Hände abgeschlagen werden, sondern die von ihnen begangenen Rechtsverletzungen sollten durch Züchtigung mit der Rute oder Peitsche geahndet werden. Auch der aus dem ersten Drittel des 13. Jahrhunderts stammende Sachsenspiegel verbot Todesurteile gegen Kinder, und ein noch nicht 12jähriger Rechtsbrecher blieb straflos. Die „Peinliche Halsgerichtsordnung“ Karls V von 1532 kannte Regelungen für Jugendliche unter 14 Jahren, die die Todesstrafe verboten und statt dessen Leibesstrafen vorsahen. Das deutsche Strafrecht unterschied bis zum Ausgang des 18. Jahrhunderts drei Altersstufen. Die Kinder im Alter von 0-6 Jahren waren nicht schuldfähig, während die Kinder im Alter von 7-14 Jahren bedingt zurechnungsfähig waren und der Richter von Fall zu Fall darüber zu entscheiden hatte, ob die Person zu bestrafen war. Die sog. „Minores“, d.h. die 14-25jährigen Täter, waren strafrechtlich in vollem Umfang verantwortlich.

Auch die Aufklarung brachte keine relevanten Neuerungen im Strafrecht in bezug auf die Behandlung junger Rechtsbrecher. Noch das Strafgesetzbuch von 1871 kannte nur einige Sonderbestimmungen für jugendliche Straftäter, die jedoch immer noch stark beherrscht waren vom Strafgedanken. Das Strafmündigkeitsalter begann mit der Vollendung des zwölften Lebensjahres, und für Täter zwischen 12 und 17 Jahren mußte das Gericht feststellen, ob der Jugendliche als strafmündig anzusehen sei. Die Strafzumessung sollte jedoch gegenüber den Erwachsenen deutlich milder sein. Nach § 57 StGB von 1871 sollte die Strafe für jugendliche Angeklagte zwischen dem gesetzlichen Mindestbetrag der angedrohten Strafart und der Hälfte des Höchstbetrages liegen. Resultat dieser Regelung waren für Jugendliche häufig kurze Freiheitsstrafen, die sie in den all- 
gemeinen Strafanstalten verbüfen mußten. Da die Haft oft nicht in Trennung vom Erwachsenenvollzug erfolgte und da der Makel des Vorbestraftseins lebenslänglich haften blieb, war diese Praxis von außerordentlicher Schädlichkeit.

Wenn bis zu dieser Zeit junge Rechtsbrecher eine mildere Behandlung bzw. Bestrafung erfuhren als Erwachsene, so war dies in der Regel in Mitleid begründet. Eine grundsätzlichere Fundierung einer besonderen Behandlung des jungen Rechtsbrechers ließ sich erst vornehmen, nachdem Kindheit und Jugend in der "Neuzeit" als besondere Phasen im Leben eines Menschen eindeutig sozial konstituiert und in ihrer besonderen Problematik auch wissenschaftlich erkannt waren (vgl. Ariès 1975), die auch ,,abweichendes Verhalten" in der Form von Rechtsbrüchen von Kindern und Jugendlichen als etwas anderes als das von Erwachsenen erscheinen ließen. Vor allem in den angelsächsischen Ländern gewann diese Sichtweise an Boden, so dab hier der Ausdruck „Delinquenz" für das rechtliche Fehlverhalten von Kindern und Jugendlichen üblich wurde, um es gegen die „Kriminalität“ der Erwachsenen abzusetzen (vgl. zur Geschichte und Ideologiekritik dieser Bewegung der „Child savers“ Platt 1969). Die internationale Reformbewegung strebte danach, den jugendlichen Rechtsbrecher nicht mehr als jungen Erwachsenen zu betrachten, sondern die biologischen, psychologischen und soziologischen Eigengesetzlichkeiten dieser Altersstufe zu berücksichtigen und entsprechend geeignete Maßnahmen zu konzipieren. Von besonderer Bedeutung war dabei der Gedanke, der bis in die Gegenwart weiterwirkt, daß die angemessene Behandlung nicht milder, sondern zweckmäßiger sein soll, was unter Umständen sogar eine härtere Behandlung erforderlich zu machen schien.

Ganz auf dieser Linie liegt die Tendenz, für Kinder und Jugendliche eine ganze Reihe von Tatbeständen als Straftatbestände zu definieren, die für Erwachsene nicht kriminalisiert sind. Dies ist bspw. im amerikanischen Strafrecht besonders ausgeprägt, in dem eine ganze Reihe von , status offenses" Auslöser strafrechtlicher Sanktionen sind. Dies gilt z.B. für den Genuß von Alkohol und Tabak, die Teilnahme an Tanzveranstaltungen in den späten Abendstunden, den vorehelichen Geschlechtsverkehr etc. Natürlich unterliegen diese Verhaltensweisen auch bei uns rechtlicher Reglementierung, doch bedient sich der Staat hier weitgehend eines anderen rechtlichen Instrumentariums bzw. sucht Problemlösungen unterhalb der Anwendung des Strafrechtes. Andererseits gibt es auch für die Bundesrepublik eine Reihe von Nachweisen dafür, daß das Strafrecht gegen Jugendliche teilweise konsequenter und härter angewendet wird als gegen Erwachsene, obwohl man doch allgemein meint, es müßte genau ungekehrt sein.

Im Jugendgerichtsgesetz von 1923 wurde in Deutschland erstmals innerhalb eines Strafgesetzbuches die Erziehung des Täters in den Vordergrund gerückt und dem Jugendrichter ein breiter Handlungsspielraum zur Verfügung gestellt (\$§ 5-7 JGG 1923), wobei schon damals ausdrücklich vorgeschrieben war, von Strafe abzusehen, wenn Erziehungsmaßregeln ausreichten. Es erfolgte die Einrichtung reiner Jugendgerichte, $J u$ gendgerichtshilfe wurde eingeführt, der Ausschluß der Öffentlichkeit gängige Praxis, und wenn Jugendstrafe unvermeidlich schien, so sollte sie doch rein erzieherisch gestaltet werden. Hier ist allerdings die Frage, inwieweit zwischen Anspruch und Realität nicht doch enorme Diskrepanzen die Regel waren.

Alle diese relativen Fortschritte wurden durch die nationalsozialistische Gesetzgebung mehr oder weniger rückgängig gemacht oder eingeschränkt. Das JGG des Jahres 1953 stellte die Erziehungsmaßregeln wieder eindeutig in den Vordergrund und hob ei- 
nige Verschärfungen und Verwässerungen der Nationalsozialisten wieder auf, konnte jedoch nicht annähernd die relativ fortschrittlichen Weimarer Ideen aufgreifen oder gar mächtig weiterentwickeln. Allenfalls war die Aufnahme der Heranwachsenden in das JGG ein Schritt von erheblicher Bedeutung. Das Gesetz aus dem Jahre 1953 hat erstaunlicherweise bis in die Gegenwart keine grundlegenden Veränderungen, sondern nur Modifikationen in kleineren Details erfahren, so daß sich die durchaus beobachtbaren Veränderungen in den justitiellen Reaktionen auf die Jugendkriminalität als eine Art ,,innerer Reform" verstehen lassen, die jedoch weit hinter dem zurückbleibt, was aufgrund sozialwissenschaftlicher Erkenntnisse hätte geschehen sollen.

\subsection{Eine Skizze der gegenwärtigen Rechtssituation im Jugendstrafrecht}

Da die justitielle Reaktion auf kriminelles Handeln junger Menschen ohne die rechtlichen Hintergründe nicht angemessen verstanden werden kann, seien einige wenige Erläuterungen zum Jugendstrafrecht gegeben. Das Jugendstrafrecht ist im Jugendgerichtsgesetz geregelt. Gemäß $\$ 1$ Abs. 1, 4 JGG erfolgt die rechtliche Einordnung der Straftaten von Jugendlichen nach den Vorschriften des allgemeinen Strafrechts, d.h. die Tat behält auch im Jugendstrafrecht ihr zentrales Gewicht und wird ohne Rückgriff auf pädagogische Kriterien juristisch klassifiziert. Wenn man das Jugendstrafrecht dennoch durch Begriffe wie ",Taterstrafrecht " und ,Erziehungsstrafrecht" kennzeichnet (Schaffstein 1983, S. 11), so liegt das darin begründet, daß das JGG einen ganzen Kanon von jugendspezifisch konzipierten besonderen Sanktionen kennt, die die Sanktionen des allgemeinen Strafrechts gänzlich ersetzen.

Die Sanktionen gehen von einfachen „Erziehungsmaßregeln“" (z.B. Erteilung von Weisungen, Erziehungsbeistandschaft, Heimerziehung) ( $\$$ 9-12 JGG), über"Zuchtmittel" (z.B. Verwarnung, Auflagen, Jugendarrest) ( $\$ \S 13-16 \mathrm{JGG}$ ) bis zu verschiedenen Formen der Jugendstrafe, wobei ausdrücklich der Strafrahmen des Erwachsenenstrafrechts nicht gilt ( $\$ 18$ Abs. $1 \mathrm{JGG}$ ). \&8 JGG läßt eine große Vielfalt von Kombinationen von Sanktionen $\mathrm{zu}$, so daß die Sanktionspraxis theoretisch ein sehr differenziertes Bild ergeben könnte. Allerdings legt $\$ 5$ Abs. 2 JGG eindeutig fest: ,Die Straftat eines Jugendlichen wird mit Zuchtmitteln oder mit Jugendstrafe geahndet, wenn Erziehungsmaßregeln nicht ausreichen“, und will damit erreichen, daß möglichst wenig eingriffsintensive Reaktionen gewählt werden, wenn mit ihnen der gewünschte Effekt erreichbar zu sein scheint. Merkwürdigerweise hat diese Regelung keine Änderung erfahren, obwohl sie doch z.B. insofern in sich widersprüchlich ist als z. B. die Heimerziehung/Fürsorgeerziehung als Erziehungsmaßregel sicher sehr viel eingriffsintensiver ist als die bloße Verwarnung, die als „,Zuchtmittel“" als die schwerere Sanktion gilt. Grundsätzlich gilt, daß im Jugendstrafrecht neben das Schuldprinzip das sog. ,Subsidiaritätsprinzip ${ }^{\text {" }}$ gestellt ist, das verlangt, daß die Rechtsfolgen primär an der Erforderlichkeit erzieherischer Maßnahmen in jedem Einzelfall zu orientieren sind. Kaiser stellt dazu fest: „Die eher tatorientierte Schuldfeststellung begrenzt sodann das Entscheidungsermessen des Gerichts hinsichtlich der Obergrenze der Intensität der angeordneten Rechtsfolge. Im Jugendstrafrecht kann weder allein um dieses Schuldausgleichs willen... gestraft werden, noch sind Maßnahmen zulässig, die zwar aus pädagogischer Sicht erforderlich, hinsichtlich der Tat jedoch unverhältnismäßig wären (Kaiser 1985, S. 174). 
Für die justitielle Bearbeitung jugendlicher Rechtsbrüche war in der Vergangenheit der Umstand sehr bedeutsam, daß in $\$ 10$ die verschiedenen Formen von Weisungen nicht abschliefiend bestimmt, sondern zum einen allgemein umschrieben und dann eher beispielhaft und nicht abschliefend aufgeführt sind. Dieser Umstand hat es ermöglicht und macht es weiterhin möglich, immer wieder neue sozialpädagogische Angebote zu konzipieren und in die Praxis einzuführen, die nach neuen Erkenntnissen positive Effekte auf straffallig gewordene Jugendliche zu haben versprechen (in der Vergangenheit bspw. Erziehungskurse, Betreuungsweisungen, Arbeitsweisungen, gemeinnützige Arbeiten, Täter-Opfer-Ausgleich etc.).

Die eher ahndenden Zuchtmittel, die nach der Devise ,,short, sharp and shocking“" wirken sollten, sind im \$ 13ff JGG abschließend aufgezählt und konnten sich daher durch die Praxis nicht wesentlich weiterentwickeln. Insbesondere der Jugendarrest, dessen Dauer auf maximal vier Wochen begrenzt ist, wird durchgehend als veraltet und unsinnig bewertet, von einigen Bundesländern praktisch gar nicht mehr angewendet und von anderen im Grunde sehr stark eingeschränkt. Die in $\$ 15$ JGG in der Fassung von 1990 umschriebenen Auflagen, die die Schadenswiedergutmachung, die Entschuldigung beim Opfer, die Erbringung einer Arbeitsleistung sowie eine Zahlung an eine gemeinnützige Einrichtung vorsehen, haben sich dagegen durchaus weiterentwickelt und scheinen an Bedeutung zu gewinnen. Interessant ist in diesem Zusammenhang, daß der sog. Täter-Opfer-Ausgleich, von dem schon bei den Weisungen die Rede war, nun auch als Reaktionsform bei den Auflagen erwähnt wird. Dies ist ein weiterer Hinweis darauf, daß dem Sanktionskanon des JGG eine zwingende pädagogische Systematik im Grunde nicht unterliegt.

Die Jugendstrafe ist die einzige im juristischen, sicher nicht im subjektiven Sinne richtige "Strafe" des JGG und darf nur als letztes Mittel eingesetzt werden. Dies erklärt, warum ihr Mindestmaß 6 Monate beträgt. Ihr Höchstmaß liegt bei 5, in Ausnahmefällen 10 Jahren ( $\$ 18 \mathrm{JGG}$ ). Für die Frage der Aussetzung der Strafe zur Bewährung gelten die Regeln des allgemeinen Strafrechtes. Gegen die Jugendstrafe werden - wie gegen die Freiheitsstrafe generell - gravierende Bedenken vorgetragen, die von der Kritik an einer unzureichenden rechtlichen Regelung des Vollzuges bis zu einer fundamentalen Kritik der Freiheitsstrafe reichen. Die Fragwürdigkeit der Freiheitsstrafe an Jugendlichen wird durch eine Reihe empirischer Arbeiten nachhaltig bewiesen (vgl. die Arbeiten in Albrecht/Schüler-Springorum 1983, insbesondere Albrecht/Lamott; Bielefeld sowie Kersten/Kreissl/von Wolffersdorff-Ehlert).

\section{Epidemiologische Befunde zur Jugendkriminalität}

\subsection{Die Entwicklung und Häufigkeit der Jugendkriminalität und der offiziellen Reaktionen im Längsschnitt}

Die Klagen über die vermeintlich immer mehr überhandnehmende Kriminalität und die besonders erschreckend erscheinende zunehmende Beteiligung junger Menschen, die zudem immer gewalttätiger zu werden scheinen, sind seit langem feststehender $\mathrm{Be}$ - 
standteil der öffentlichen Meinung und zahlreicher Berichte in den Medien auch in der Bundesrepublik Deutschland, obwohl die vorhandenen Daten eigentlich eine etwas andere Sprache sprechen. Zunächst einmal muß man, aus historischer Perspektive gesehen, die Behauptung, in Deutschland nehme die Gewaltkriminalität insgesamt seit langem zu, deutlich zurückweisen. Verglichen mit den Verhältnissen in der zweiten Hälfte des vorigen Jahrhunderts und den ersten Jahrzehnten dieses Jahrhunderts ist diese Entwicklung (langfristig betrachtet) entschieden rückläufig. Das heißt nicht, daß es kurzfristig nicht doch zu scheinbar erheblichen Steigerungen kommen kann bzw. in jüngster Zeit auch gekommen ist, doch muß beachtet werden, daß gerade bei besonders schweren Delikten, an denen sich die öffentliche Diskussion leicht entzündet, die Häufigkeitsziffern wegen ihrer sehr niedrigen Höhe durch vergleichsweise geringe Steigerungen der absoluten Fallzahlen exorbitante Steigerungsraten aufweisen können, die zu völlig falschen SchluBfolgerungen führen können.

Faßit man nun speziell die Kriminalität der Jugendlichen und der Heranwachsenden ins Auge und betrachtet einmal die Verhältnisse und die Entwicklungen in den 70er Jahren, für die wir über eine methodenkritische Studie verfügen (Albrecht/Lamnek 1979), so sollten wir einige wenige Fakten zur Kenntnis nehmen, die das Problem der ,Jugendkriminalität" auf seine wirklichen Dimensionen zurückführen. Nach Albrecht/Lamnek (1979, S. 163) wurden im Jahre 1971 von der gesamten 6- bis unter 2ljährigen Wohnbevölkerung der Bundesrepublik 2,5\% $(\mathrm{KBZ}=2517)$ und $19773,1 \%(\mathrm{KBZ}=3067)$ von der Polizei als einer Straftat verdächtig registriert. Damit wird deutlich, daß die Kriminalität der jungen Menschen in den 7 er Jahren zwar ein nicht zu verkennendes soziales Problem darstellte, das jedoch im Grunde zu hysterischen Reaktionen keinen Anlaß gab.

Zwar ist die Zahl der bekanntgewordenen Straftaten insgesamt (also für alle Altersgruppen und Delikttypen) pro 100000 Einw. in den späten 70er und den frühen 80er Jahren deutlich gestiegen, aber seit dieser Zeit ist sie eindeutig stagnierend (ca. 7000 pro 100000 Einw.). Geht man in die Details, so zeigt sich bei der Verhältniszahl der strafmündigen Tatverdächtigen für die männlichen Jugendlichen, daß diese zwar in den 70 er Jahren bis etwa 1982 gestiegen ist (von ca. 7400 auf ca. 8800), aber seit dieser Zeit ist sie zunächst stark rückläufig gewesen und bewegt sich zuletzt auf einem Niveau von ca. $6300 \mathrm{im}$ Jahre 1988. Ein ganz ähnliches Bild - auf etwas höherem Niveau - ergibt sich bei den männlichen Heranwachsenden. Die Daten für die weiblichen Jugendlichen und Heranwachsenden weichen davon deutlich $a b$. Grundsätzlich beträgt die Häufigkeitsziffer der weiblichen Jugendlichen nur etwa ein Drittel der männlichen Jugendlichen (bei den Heranwachsenden sogar nur etwa ein Sechstel), aber auch hier gab es seit den 70er Jahren zunächst deutliche Steigerungen (von ca. 1400 im Jahre 1972 auf ca. 2000 im Jahre 1982 bei den weibl. Jugendlichen), denen dann nur ein schwacher Rückgang bis zu den späten 80er Jahren folgte. Diese Daten sprechen ganz eindeutig gegen die Behauptung einer dramatischen Zuspitzung der Jugendkriminalität; eher das Gegenteil ist der Fall. Wechselt man von der Polizeilichen Kriminalstatistik zur Gerichtsstatistik, die wegen der eindeutigeren rechtlichen Würdigung der Straftaten einerseits genauer, wegen ihrer größeren Selektivität jedoch theoretisch weniger aussagefähig ist, und spannt einen zeitlich weiteren Bogen, so sprechen auch hier die Verhältniszahlen eine eindeutige Sprache. Die Verurteiltenziffern bei den Jugendlichen insgesamt steigen zwar von 1588 im Jahre 1968 auf 2068 im Jahre 1982, aber bis zum 
Jahre 1986 sind sie bspw. wieder auf 1532 gesunken (recht ähnliches Bild bei geschlechtsspezifischer Betrachtung). Für die Heranwachsenden insgesamt ergibt sich ein ähnlicher Verlauf - auf deutlich höherem Niveau -, der jedoch insgesamt für diesen Zeitraum ein Absinken der Verurteiltenziffer von 3252 auf ca. 2700 aufweist (bei den weiblichen Heranwachsenden bleibt bei zwischenzeitlich deutlichem Anstieg das Niveau der späten 60er Jahre erhalten). Auch hier also keinerlei Beleg für eine besondere Zuspitzung im Bereich der Jugendkriminalität, sondern eher das gegenteilige Bild. Dabei ist zu betonen, daB die von manchen Autoren (vgl. Adler 1975, 1981) erwartete bzw. behauptete Aufholbewegung der Frauen im Bereich der Kriminalität als Begleiterscheinung der Emanzipation offensichtlich nur begrenzt eingetreten ist (vgl. H.-J. Albrecht 1987; Albrecht/Howe/Wolterhoff-Neetix 1988; Steffensmeier 1978; Steffensmeier/Steffensmeier 1980).

Absolut gesehen ergeben sich daraus natürlich immer noch beachtenswert viele Fälle, in denen die gesellschaftlichen Kontrollinstanzen zum Strafrecht greifen zu müssen glauben, um dem abweichenden Verhalten von Jugendlichen Einhalt zu gebieten. Betrachtet man dazu absolute Zahlen, so wurden 1977 in ca. 100000 Jugendgerichtsverfahren Urteile gefällt, während dies im Jahre 1987 bspw. immer noch ca. 67000 waren. Betrachtet man die dabei ergangenen Sanktionen, so sind die relativen Anteile der drei Typen „Erziehungsmaßregeln“ (37,1\%), „Zuchtmittel“ (56,4\%) und „Jugendstrafe“ $(6,45 \%)$ insofern im Wandel als zwar der Anteil der Jugendstrafen in diesen 10 Jahren völlig konstant, aber der Anteil der Erziehungsmaßregeln (von ca. 24 auf ca. $37 \%$ ) auf Kosten der Zuchtmittel deutlich expansiv ist. Das kann man so deuten, daß die Jugendgerichtsbarkeit anscheinend da, wo sie gewisse Spielräume sieht, auch davon Gebrauch macht und die weniger eingriffsintensiven Sanktionen wählt. Ob sie dabei wirklich weit genug geht, ist allerdings eine weitere Frage. Immerhin ergingen im Jahre 1987 in 4339 Verfahren gegen Jugendliche Verurteilungen zu Jugendstrafen, und damit zu einer Freiheitsstrafe, deren zweifelhafte Wirkung eigentlich nicht mehr zweifelhaft ist. Dabei ist ferner zu beachten, daß jeweils ca. 30\% dieser Jugendstrafen für Jugendliche (Heranwachsende sind hier nicht berücksichtigt) auf mehr als 1 Jahr lauten.

Bezieht man nun noch das Wissen mit ein, daß die Zahl der der Polizei bekannt gewordenen Straftaten und der Straftäter in hohem Maße eine Funktion der Anzeigebereitschaft der Bevölkerung ist (ca. $80 \%$ der polizeilichen Ermittlungsverfahren gehen auf private Anzeigen zurück) und daß wiederum diese Anzeigebereitschaft gegenüber dem abweichenden Verhalten von jungen Leuten aus bestimmten Gründen zugenommen zu haben scheint (vgl. Albrecht/Lamnek 1979), so könnte es sein, daß die „Kriminalität" der Jugendlichen in Wirklichkeit gar nicht gestiegen oder konstant geblieben ist, sondern de facto sogar ganz entschieden zurückgegangen ist und in der Gegenwart nur anders als früher gedeutet und ,problematisiert" wird (vgl. dazu unten den sog. Labeling approach).

\subsection{Ausgewählte epidemiologische Befunde zur aktuellen Situation}

Unser gegenwärtiges Wissen reicht also nicht aus, um zu entscheiden, welche dieser Deutungen die richtige ist. Eine solche Aussage wäre nur dann möglich, wenn wir im 
historischen Langsschnitt Kriminalitätsdaten zur Verfügung hătten, die nicht durch diese komplexen Selektionsfilter von informellen und formellen Kontrollinstanzen produziert bzw. deformiert sind (vgl. dazu Kerner 1972; Sack 1968; Sack 1978). Dazu könnten sog. „Self-Report-Daten" beisteuern, die seit einiger Zeit erhoben werden und zu sehr wichtigen Ergebnissen geführt haben. Eine der Schlüsselentdeckungen für die moderne Kriminalitätsforschung beruht auf solchen Daten (Nye/Short/Olson 1958; Short/Nye 1968). So zeigte sich, daß die auf der Basis offizieller Kriminalstatistiken immer wieder behauptete negative Korrelation zwischen Schichthöhe und Kriminalitätsbelastung, die den Ausgangspunkt vieler theoretischer Erklärungsansätze abgibt, nicht existiert, wenn man diese Datenquelle heranzieht (vgl. dazu vor allem die zentrale Arbeit von Tittle/Villemez/Smith 1978), sondern daß man von einer annähernden schichtmäßigen Gleichverteilung ausgehen muß. Nun zeichnet sich die Forschung auf der Basis der Self-Report-Daten dadurch aus, daß sie in der Regel allenfalls episodisch, bei kleineren, oft sehr selektiven und systematisch verzerrten Auswahlen durchgeführt wurde, so daß es nur selten möglich ist, auf der Basis dieser Daten Entwicklungen im Längsschnitt zu rekonstruieren oder Vergleiche zwischen verschiedenen Regionen oder sonstigen sozialen Einheiten anzustellen. Zwar verfügen wir nach den Studien von Quensel (1971), Brusten/Hurrelmann (1973) über einige andere lokale Studien, doch sind diese jeweils nur zu wenigen Zeitpunkten und an begrenzten Stichproben durchgeführt worden, so daß wir praktisch keine Entwicklungstrends aus diesen Daten ableiten können.

Dennoch haben diese Studien insofern einen großen Wert für die Kriminalitätsforschung als sie Dunkelfelddaten liefern, die den ,wahren“" Verhältnissen eher entsprechen als die Daten der Kontrollinstanzen. Um eine Vorstellung von der ,wahren“" Kriminalitätsbelastung der Jugend zu vermitteln, stellen wir einige wenige Daten aus den Untersuchungen von Albrecht u.a. dar (Albrecht/Howe/Whlterhoff-Neetix 1988; Albrecht /Howe/Wolterhoff 1991; Albrecht / Howe 1991), die aus einer für die Städte (mit Umland) Bielefeld und Münster repräsentativen Stichprobe aus dem Jahre 1986 stammen, ohne auf Details der Untersuchungsmethode eingehen zu können. Die Untersuchung erstreckte sich auf 13-17jährige, die anhand einer Umschreibung von 12 Deliktformen angeben konnten, ob sie eine entsprechende Handlung schon einmal in ihrem Leben angegeben haben bzw. wie oft dies im letzten Jahr schon einmal vorgekommen ist. Auf die vorgegebenen Delikttypen entfallen in der offiziellen Kriminalstatistik in der Regel ca. $75 \%$ aller Delikte, so daß unsere Auswahl geeignet gewesen sein müßte, die ganz überwiegende Mehrzahl der Delikte zu erheben. Für die gesamte Lebenszeit betrachtet behaupten ca. $40 \%$ der Probanden, daß sie keine der genannten kriminellen Handlungen begangen hätten (im Detail für die Oberkategorien Betrug ca. $65 \%$, einf. Diebstahl $68 \%$, Körperverletzung $75 \%$, Sachbeschädigung $78 \%$, schwerer Diebstahl $88 \%$ ohne Delikt), doch ist dabei zu beachten, daß diese Daten nach Alter und Geschlecht der Probanden stark variieren. So behaupten nur ca. 34 \% der Jungen, sie hätten keine Delikte begangen, und die Delikthäufigkeit steigt signifikant mit dem Alter. Insbesondere wird deutlich, daß die Zahl derjenigen Jugendlichen, die mehrfach delinquent gehandelt hat, mit dem Alter ansteigt. Zwischen den Geschlechtern zeigen sich ganz deutliche Disparitäten, die umso größer werden, je schwerer die Delikte sind (ganz entsprechend der internationalen Literatur; vgl. Steffensmeier a.a.O.). Für andere wichtige Details sei auf die entsprechenden Studien verwiesen. Insgesamt zeigen die Daten, 
daß wir davon ausgehen müssen, daß bis zum Erreichen des Status des Heranwachsenden bzw. des jungen Erwachsenenalters nahezu jeder Mensch Handlungen begangen hat - wenn auch meist im Dunkelfeld verbleibende -, die potentiell kriminalisierbar sind. So gesehen stimmte die Behauptung einiger wichtiger Theorien (vgl. z.B. Sack 1968; Sack 1978), daß Kriminalität ein universell verbreitetes Phänomen darstellt, das allerdings für verschiedene gesellschaftliche Subgruppen mit unterschiedlichen Kriminalisierungsrisiken verknüpft ist. Dieser Befund ist deshalb von besonderer Bedeutung, weil er allen dramatisierenden Reaktionen auf Jugendkriminalität den Boden entzieht. Offensichtlich betätigt sich ein erheblicher Teil aller Menschen in der Jugend in einem nicht unerheblichem Maße kriminell, ohne entdeckt zu werden, und offensichtlich werden aus fast allen diesen „Delinquenten“ später doch mehr oder weniger gesetzestreue Bürger. Die von den Kontrollinstanzen nahezu durchgehend vertretene Meinung, daß ohne ihre Intervention (sei sie pädagogisch oder strafend) aus dem jugendlichen „Kriminellen" ein "Karriere-Krimineller" werde, erhält dadurch einen ersten schweren Schlag, dessen Wucht noch zunimmt, wenn man behauptet, daß gerade diese Intervention meist erst die Entwicklungsaussichten verschlechtert (vgl. Albrecht 1990), und dies umsomehr, je intensiver diese Intervention ausfällt (vgl. McCord 1990). Ob die Daten allerdings die Behauptung rechtfertigen, Kriminalität sei zwischen den gesellschaftlichen Gruppen, insbesondere zwischen den sozialen Schichten gleich verteilt, soll an anderer Stelle erörtert werden.

\section{Ausgewählte Theorieansätze zur Erklärung von Jugendkriminalität und einige Forschungsbefunde}

Betrachten wir die Geschichte der Theorien zur Erklärung von Kriminalität, so stellen wir fest, daß die unabhängigen, also die erklärenden Variablen, von der biologischgenetischen, physiologischen, individualpsychologischen, sozialpsychologischen, mikrosoziologischen bis zur makrosoziologischen Ebene reichen. Lange Zeit waren die theoretischen Erklärungsansätze insofern unspezifisch als sie Kriminalität insgesamt zu erklären bemüht waren und nicht die Besonderheit des kriminellen Verhaltens von Jugendlichen beachtet haben. Allerdings kann man dies auch als einen relativen Vorteil ansehen, da natürlich grundsätzlich die allgemeinere Theorie, die sich auf eine breitere Palette von Phänomenen anwenden läßt, wertvoller ist. Es könnte sich jedoch herausstellen, daß jene Theorien, die auf der Basis des Wissens über die Kriminalität von Erwachsenen entwickelt wurden, nicht auf Jugendliche übertragbar sind etc. In den letzten Jahrzehnten befassen sich die meisten Theorien kriminellen Verhaltens jedoch eher mit dem Verhalten Jugendlicher bzw. Heranwachsender oder junger Erwachsener, so daß sich das obige Problem eigentlich nicht mehr stellt. Anders verhält es sich mit der Variable Geschlecht, da die empirische Forschung, und damit auch die Theoriebildung, aufgrund der für die meisten Gesellschaften immer wieder berichteten entschieden höheren offiziellen Kriminalitätsbelastung (gemessen durch die üblichen Häufigkeitsziffern bzw. Belastungsziffern der Polizeilichen Kriminalstatistik, der Gerichtsstatistik oder der Strafvollzugsstatistik) der männlichen Bevölkerung ihr Augenmerk fast nur auf das 
männliche Geschlecht gerichtet hat. Hinzu kommt, daß die Jugendkriminalität sich traditionell in den großen städtischen Gebieten in besonders prägnanter Weise präsentiert und daß sich wegen der aus ganz bestimmten Gründen besonderen Ausprägung des Kriminalitätsproblems in den USA gerade in diesem Land die Forschung dort ungeheuer stark entwickelt hat, so daß man fast versucht ist zu sagen, unser theoretisches und empirisches Wissen über Kriminalität sei in Wirklichkeit Wissen über das kriminelle Handeln männlicher Jugendlicher in den großen Metropolen der Vereinigten Staaten, dessen Übertragung auf das weibliche Geschlecht, andere Altersgruppen, die ländliche Bevölkerung, ja auf andere gesellschaftliche Konstellationen durchaus fragwürdig ist. Mit diesem Vorbehalt sei ein äußerst knapper Überblick über einige zentrale Theorien der Jugendkriminalität gegeben.

Dabei verzichten wir auf die Einhaltung disziplinärer Grenzen und vermeiden jegliches Vollständigkeitsstreben, da dies nie einzulösen wäre. Wir verzichten auch auf eine Darlegung biologisch-genetischer und/oder physiologischer Erklärungen, nicht deshalb, weil wir davon ausgehen, daß sie grundsätzlich ohne irgendeinen wissenschaftlichen Wert seien, sondern deshalb, weil sie ganz offensichtlich ungeeignet sind, die Kriminalität von Jugendlichen als eines Massenphänomens, das sozusagen jeden betriff, verständlich zu machen. Bestimmte, aber in der Regel relativ seltene Fälle kriminellen Handelns von Jugendlichen mögen in einer genetischen Unregelmäßigkeit/Störung ihre Ursache haben, oder bestimmte problematische Ernährungsgewohnheiten mögen bei einem sehr kleinen Teil der Jugendlichen für bestimmte Kumulationen von Auffälligkeiten kausal bedeutsam sein, aber für $99 \%$ aller Fälle sollte all das irrelevant sein, so daß wir auf eine nähere Diskussion verzichten können sollten (für umfassendere Darstellungen empirischer Forschungsergebnisse vgl. Albrecht 1982; Amelang 1986).

\subsection{Der Psychologie nahestehende Ansätze}

\subsubsection{Psychoanalyse}

Klassische Beiträge zur Kriminalitätserklärung hat die Psychoanalyse beizusteuern, die Kriminalität als Ausdruck einer Persönlichkeitsstörung deutet, deren Bedingungen in frühen Beeinträchtigungen der psychischen Entwicklung gesucht werden (z.B. traumatische Veränderungen oder Defekte der sozialen Umwelt). Auch wenn Psychoanalytiker grundsätzlich die Möglichkeit nicht leugnen, daß auch bei ,normaler"“ Persönlichkeitsstruktur bei extremer aktueller Belastung der verschiedensten Art Kriminalität auftreten kann, so haben sie doch eher zwei Delinquenzkonzepte entwickelt, die auf bestimmte Persönlichkeitsstrukturen als Quelle abweichenden Verhaltens abstellen. Ergeben sich im Verlaufe der Sozialisation ungelöste Konflikte zwischen verdrängten Triebansprüchen des Es und einem zu starken Überich, das u.U. auf rigide elterliche Erziehungs- oder Unterwerfungsprozesse zurückgeht, so kommt es u.U. zur neurotisch bedingten Kriminalität, die auch als ,,Ausagieren" gedeutet wird, während andere Neurotiker sich in der Symptomwahl z.B. gegen die eigene Person richten. Erfolgt dagegen durch gravierende Störungen der frühen Mutter-Kind-Beziehung, die eine Identifikation mit der Mutter und eine Gewissensbildung verhindert oder behindert haben, die Ausbil- 
dung eines zu schwachen Über-Ichs, so muß mit durch Verwahrlosung bedingter Kriminalität gerechnet werden. Die wichtige Sozialisationstechnik des Liebesentzuges, die die Grundlage für das Lernen von Schuldgefühlen ist, bleibt in solchen Fällen relativ wirkungslos, denn da die intensive mütterliche Zuwendung nicht erfahren wurde, stellt ihr Entzug auch keine relevante Entbehrung mehr dar.

Neben diesen älteren Konzeptionen gibt es umfassendere Modelle eines psychoanalytischen Phasenmodells der Entstehung psychischer Störungen und Erkrankungen, durch das neurotische und verwahrlosungsbedingte Delinquenzformen kausal auf Entwicklungsstörungen in bestimmten Altersabschnitten der Kindheit zurückgeführt werden (vgl. Toman 1983). Während die Theorie des ,dissozialen Syndroms" (De Boor 1977) vor allem die Bedeutung von Über-Ich-Defiziten betont, stellen andere Autoren stärker auf Probleme des Realitatskonzeptes bzw. Ich-Störungen ab, die sich bei den Individuen in mangelnder Frustrationstoleranz, mangelnder Konfliktbewältigungsfähigkeit, begrenzten Zeit-und Planungshorizonten, Fehlattributionen sowie unangemessenen Rationalisierungstendenzen auswirken und daher die Wahrscheinlichkeit kriminellen Handelns deutlich erhöhen (Redl/Wineman 1951).

Eine grundsätzliche wissenschaftstheoretische Kritik der psychoanalytischen Theorie kann hier unterbleiben. Auch wenn die Frage der empirischen Haltbarkeit vieler Aussagen durchaus skeptisch beurteilt werden muß, so kann nicht bestritten werden, daß die Bedeutung familialer Deprivationen, psychischer Konflikte, irrationaler Motive für die Erklärung abweichenden Verhaltens verschiedenster Art ernst genommen werden muß. Nachhaltig muß jedoch davor gewarnt werden, allzu vorschnell kriminelles Verhalten kausal auf bestimmte Persönlichkeitsmerkmale zurückzuführen, da eine solche Strategie mit der Universalitat kriminellen Handelns nicht vereinbar sein würde. Desgleichen gerät die psychoanalytische Position zu schnell auf eine Bahn, die Kriminalitätsphänomene zu sehr individualisiert und pathologisiert, obwohl doch die soziale und kulturelle Relativität der Normen, gegen die kriminelles Handeln verstößt, auf der Hand liegt.

\subsubsection{Die Theorie der moralischen Entwicklung und der Rechtssozialisation}

Ein in den letzten Jahren besonders bekannt gewordener ,kognitiver“ Ansatz, der sich für die Entwicklung einer Theorie der Kriminalität eignen zu können schien, ist in der „Entwicklungstheorie des moralischen Urteils" von Kohlberg (1971) zu sehen, die auf einer Weiterentwicklung Piagetscher Ideen beruht. Kohlberg nimmt an, daß die Entwicklung des moralischen Urteils von der Entwicklung des Denkens (operativen Niveaus) und der Entwicklung sozial-kognitiver Kompetenzen (z.B. zur Perspektivenübernahme) abhängt. Kohlberg nimmt eine Entwicklungssequenz an, die als invariant und als universell gültig verstanden wird. Sie kennt drei Niveaus, die in sich jeweils zwei Stufen darstellen, wobei die letzte Stufe - empirisch gesehen - selten erreicht wird. Das sogeannte ,vorkonventionelle Niveau“ kennt auf der ersten Stufe die „Orientierung des Akteurs an Gehorsam und Strafe" und auf der zweiten Stufe die sog. ,, naivegoistische Orientierung", die sich dadurch auszeichnet, daß Handlungen nach ihrem instrumentellen Wert zur Befriedigung eigener Bedürfnisse bewertet werden. Stufe 3, als die untere Stufe des ,konventionellen Niveaus", ist gekennzeichnet durch die Orien- 
tierung am Bild des „,braven Kindes“, d.h. wichtig sind die Erwartungen anderer, während auf der Stufe 4 die „Orientierung an Autorität und sozialer Ordnung” bezeichnend ist. Auf der unteren (5.) Stufe des nachkonventionellen oder prinzipiengeleiteten Niveaus orientieren sich die Akteure an Recht und Sozialverträgen, deren Willkürlichkeit erkannt, aber doch als durch die Mehrheit vertretene Grundlage akzeptient werden. Auf der 6. Stufe, der der sog. Prinzipienorientierung, basieren die Handlungsbewertungen auf universellen Prinzipien der Gerechtigkeit, die das Individuum in freier, vernünftiger Entscheidung wählt (vgl. als allgemeine Darstellungen zu diesem Theoriegebäude Kohlberg 1969; 1971; 1976; 1981; 1984; sowie Tapp/Kohlberg 1976).

Sehen wir von einigen fundamentalen methodischen Problemen der Handhabung der Forschungsoperationen ab, die zur Feststellung der moralischen Entwicklung von Individuen durchgeführt werden müssen, sondern konzentrieren uns auf die Frage des Bezuges dieser Entwicklungsstufen zur Jugendkriminalität, so ist klar, daß sich Bezüge zur Rechtssozialisation (vgl. dazu den interessanten Theorievergleich zwischen dem Ansatz Kohlbergs und der Lerntheorie aus der Tradition von Akers u.a. sowie die empirischen Befunde bei Cohn/White 1990) aufdrängen. Verlaufen Sozialisationsprozesse so, daB Probanden überhaupt nicht oder entwicklungsverzögert das konventionelle Niveau erreichen, so wäre mit einer deutlichen Erhöhung der Wahrscheinlichkeit kriminellen Handelns zu rechnen. Erreichen Jugendliche nur die Stufe drei, so wäre zu befürchten. $\mathrm{da \beta}$ sie sich weniger von den abstrakten Rechtsnormen, sondern eher von den antizipierten Erwartungen anderer, z.B. von peers, in potentiell devianten Jugendgruppen leiten lassen und damit der Gefahr, kriminell zu handeln, verstärkt ausgesetzt sind. Problematischer wird es schon, zwischen den erwartbaren Auswirkungen der Verortung auf der vierten bzw. 5 . Stufe für das Legalverhalten zu unterscheiden. Vorstellbar wäre bspw., daß in bestimmten sozialen Situationen eine konsequente Orientierung auf der fünften Stufe die Wahrscheinlichkeit von Brüchen des konkret existierenden positiven Rechtes erhöht. Dies wird jedoch ganz zweifellos zu einem Problem auf der Stufe 6, der der Prinzipienorientierung, denn bei gegebener gesellschaftlicher Ordnung und gegebenem Recht mag eine konsequente Prinzipienorientierung auf die Begehung von Rechtsbrüchen hinauslaufen. Wie dem auch immer sei. Empirische Überprüfungen der Annahme, daß Kriminalität mit einem niedrigeren Niveau der Moralentwicklung korreliert, ergeben durchaus widersprüchliche Ergebnisse (vgl. Irvine 1979; Schumann 1985; Cohn/White 1990). Dies dürfte nicht zuletzt darin begründet liegen, daß diese Theorie zum einen die emotionalen Faktoren allzu sehr vernachlässigt, vor allem aber relativ unbegründet unterstellt, die Befähigung zu einem bestimmten Niveau der moralischen Reflektion sei bedeutungsgleich mit der Anwendung dieser Kompetenz im tatsächlichen Verhalten, ganz abgesehen davon, daß Handeln immer in bestimmten Situationen erfolgt, die hier völlig außer Betracht bleiben. 


\subsection{Soziologisch orientierte ätiologische Erklärungsansätze}

\subsubsection{Die Theorie der differentiellen Assoziation}

Eine der klassischen Theorien der Erklärung von Kriminalität allgemein stammt von Sutherland (1968 bzw. 1939), der sich in seinen Ideen sehr stark an der Lerntheorie orientiert hat, die er jedoch in einer relativ groben Form entwickelte, wobei die Zuordnung seines Beitrages zur Kriminalpsychologie oder Kriminalsoziologie hier nicht näher diskutiert werden muß. Der entscheidende Grundgedanke der neun Hypothesen Sutherlands, die in sich wenig stimmig und teilweise widersprüchlich, teilweise redundant sind, besteht darin. daß abweichendes und kriminelles Verhalten wie jedes Verhalten erlerntes Verhalten ist. Für die Frage, ob ein Individuum kriminelles oder nichtkriminelles Verhalten zeigt, ist entscheidend, ob es in seiner mikrosozialen Umwelt einen Überschuß an Definitionen erlernt hat, die kriminelles Verhalten positiv bewerten und damit zulassen. Für den Lemprozeß ist besonders wichtig, welche interpersonellen Kontakte ein Individuum aufweist und welche Definitionsleistungen mit diesen Kontakten verknüpft sind. Je früher im Leben eine Person in Kontakt mit kriminellen Definitionsleistungen kommt, desto größer ist die Wahrscheinlichkeit, daß sie später selbst kriminell handelt. Je intensiver die Kontakte zu kriminellen Situationsdefinitionen sind, desto größer ist die Wahrscheinlichkeit, daß eigenes kriminelles Handeln daraus resultiert etc. Sutherland erklärt die Häufigkeit kriminellen Verhaltens als direkten Niederschlag dieser differentiellen Kontakte. Dabei ist natürlich auffällig, daß Sutherland die Verteilung solcher, Kriminalität positiv bewertender Situationsdefinitionen in der Gesellschaft selber nicht erklärt und auch die Entstehung der differentiellen Assoziationen nicht eigentlich thematisiert. So gesehen argumentiert er eher psychologisch als soziologisch, ganz abgesehen von einer ganzen Reihe von Vagheiten, auf die die Literatur immer wieder hingewiesen hat (vgl. Opp 1974). Auf der anderen Seite ist ganz eindeutig, daß seine Erklärungsmechanismen sehr gut auf die moderne Lerntheorie zurückzuführen sind, wie es verschiedene Autoren (Akers u.a. 1979; Akers 1985) auch wiederholt versucht haben. Im übrigen ist deutlich zu erkennen, daß diese Sutherland'sche Theorie sich als noch unzulänglicher Vorläufer der sozial-kognitiven Lerntheories Banduras (1979) deuten läßt, die deshalb mit Sicherheit bei einer Weiterentwicklung dieses Ansatzes zu berücksichtigen ist. Eine Reihe von neueren empirischen Arbeiten hat die Brauchbarkeit des Ansatzes der differentiellen Assoziation durchaus belegen können (vgl. vor allem Akers u.a. 1979), auch wenn sich bei genauerer Betrachtung zeigt, daß diese Autoren - teilweise uneingestanden - recht zahlreiche erklärende Variablen aus anderen Theorien (z.B. labeling approach u. Subkulturtheorie) einbeziehen und damit die Ursprungstheorie stark verwässern.

\subsubsection{Die Anomietheorie}

Stellte die Theorie der differentiellen Assoziation von Sutherland schon einen großen Fortschritt dar, weil sie darauf verwies, daß abweichende und kriminelle Dispositionen erlernt werden und nicht quasi naturwüchsig gegeben sind, so ergaben sich die größeren 
wissenschaftlichen Fortschritte im Bereich der Kriminologie in den Jahrzehnten nach Sutherland doch eher im Bereich der Kriminalsoziologie. Hier ist an worderster Stelle die Anomietheorie Robert K. Mertons zu nennen, die sich darum bemüht. die gesellschaftliche Verteilung abweichenden Verhaltens auf grundsätzliche sozialstrukturelle Gegebenheiten zurückzuführen (vgl. als beste Einführung und theoretische und empirische Wertung dieser Theorie Bohle 1975 sowie Lamnek 1983). Merion nimmt seinen Ausgangspunkt von der Beobachtung, daß in bestimmten Gesellschaften die kulturelle und die soziale Struktur nicht in idealer Weise aufeinander abgestellt sind, sondern teilweise dissoziiert sein können. Betont eine Gesellschaft bestimmte zentrale kulturelle Werte derart entschieden, daß sie von allen Subjekten der Gesellschaft im Sozialisationsprozeß äußerst stark internalisiert werden, stellt jedoch durch die Gestaltung des Sozialisationsprozesses nicht sicher, daß die Individuen mit der gleichen Intensität auch verinnerlichen, welche Mittel und Wege zur Erreichung dieser Ziele als legitim angesehen werden, so ergibt sich insbesondere dann eine sehr prekäre Situation, wenn die Chancen, auf legale Art und Weise die allgemein geteilten Werte und Ziele zu erreichen, nicht gleich verteilt sind. In dieser Situation des ,, strains" stehen dem Individuum bzw. bestimmten gesellschaftlichen Gruppen bestimmte Formen der Anpassung zur Verfügung, von denen die sog. ,Innovation" eine solche ist, bei der zwar die allgemein geteilten Ziele und Werte beibehalten, die bisher als legitim angesehenen Mittel zur Erreichung dieser Ziele jedoch durch andere, ,innovative" Lösungen ersetzt werden. Andere Anpassungsformen zeichnen sich dadurch aus, daß die Individuen die allgemein geteilten Ziele aufgeben, aber an den als legitim angesehenen Mitteln zur Erreichung dieser Ziele festhalten (Ritualismus); oder dadurch, daß sie sowohl in bezug auf die Ziele als auch in bezug auf die Mittel auf Distanz gehen (Rückzug) oder daß sie sowohl die Ziele als auch die als legitim angesehenen Mittel aktiv durch andere Ziele und Mittel zu ersetzen versuchen (Rebellion). Merton glaubte, durch Hinweise auf die Raten abweichenden Verhaltens in verschiedenen gesellschaftlichen Gruppen zeigen zu können, $\mathrm{da} ß$ es tatsächlich zwischen dieser Dissoziation von kultureller und sozialer Struktur auf der einen Seite, die sich als Zustand der Anomie beschreiben läßt, und der Verteilung abweichenden Verhaltens auf der anderen Seite deutliche Beziehungen gibt.

Diese auf den ersten Blick bestechende makrosoziologische Theorie des abweichenden Verhaltens weist einige gravierende Mängel auf (vgl. jedoch auch Merton 1964), die sich insbesondere dann erkennen lassen, wenn man sich die Frage stellt, ob die Unterstellung, daß in modernen differenzierten Gesellschaften alle gesellschaftlichen Teilgruppen die zentralen Ziele und Werte der Gesellschaft wirklich teilen und in der gleichen Intensität verinnerlichen, stimmen kann. Tatsächlich gibt es zahlreiche Hinweise darauf, daß die höchsten Ziele und Werte sowohl qualitativ als auch quantitativ zwischen einzelnen Teilgruppen variieren, so daß sich die Anomietheorie in dieser einfachen Form mit Sicherheit nicht aufrecht erhalten läßt. Insbesondere scheint es angemessen, zwischen Aspirationen und Erwartungen zu unterscheiden, um angemessen differenzierende Aussagen machen zu können. Dadurch, daß sich die bei Merton unterstellte negative Korrelation zwischen Schichtzugehörigkeit und Delinquenzhäufigkeit in einer Vielzahl von Studien nicht zeigen ließ, ergibt sich von vornherein auch ein erheblicher Zweifel an der gesamten Konzeption Mertons, der durch viele empirische Analysen im Detail bestätigt wird (vgl. die Befunde in Clinard 1964). Mit Bezug auf die Frage des Zusammenhangs zwischen Schichtzugehörigkeit und Kriminalität hat Tittle (1983) aber 
auch nahezu allen anderen Kriminalitãtstheorien immanente Inkonsistenzen nachweisen können, die Anlaß zur Revision nahezu aller Ansätze sein sollten. Da wundert es nicht. wenn sich empirisch ergibt, daß der Zusammenhang zwischen Schicht und Kriminalitätshäufigkeit nicht nur global nicht nachweisbar ist (Tittle/Villemez/Smith 1978), sondern auch dann im Grunde nicht, wenn man die verschiedensten in den Theorien vorgeschlagenen Spezifikationen dieser Relation berücksichtigt (vgl. Tittle/Meier 1990). Auch in der Bundesrepublik zeigen sich auf der Basis won Self-Report-Daten nur für ganz spezifische Delikte und soziale Untergruppen entsprechende, meist inhaltlich sehr schwache Zusammenhänge (vgl. Albrecht/Howe 1991). Andererseits ist zu beachten, daß ein Teil der Kritik an den Strain-Theorien aus der Sicht der Kontrolltheorie (s.u.) fragwürdig ist (vgl. Bermard 1984) und viele der empirischen Überprüfungen der Menonschen Anomietheorie von einer falschen Methodologie ausgehen (vgl. Bernand 1987). Nach Jahren der Stagnation scheint wieder Leben in die Anomietheorie zu kommen, zumal sich zeigen läßt, daß makrosoziologische Studien, die Anleihen bei der Anomietheorie machen, passable Erklärungsleistungen erbringen (vgl. Albrecht/Howe 1992). Eine Wiederbelebung dieser Debatte kann man nur begrüßen, da Analysen auf der gesamtgesellschaftlichen Ebene seit langer Zeit selten geworden sind.

\subsubsection{Die subkulturtheoretischen Ansätze}

Haben wir gerade darauf hingewiesen, daß die Merton sche Unterstellung eines allgemeinen Wertekonsenses durchaus zweifelhaft sein dürfte, so betonen bestimmte Versionen der Subkulturtheorie genau diesen Aspekt der Unterschiedlichkeit verschiedener Teilkulturen in der modernen Gesellschaft. So vertritt beispielsweise Miller (1968) die These, daß die in bestimmten westlichen Gesellschaften historisch seit langem gewachsene Unterschichtkultur sich dadurch auszeichnet, daß sie bestimmte Kristallisationskerne aufweist, die sich so darstellen, daß Jugendliche aus dieser unterschiedlichen Kultur dann, wenn sie sich den in dieser Subkultur geltenden Erwartungen gegenüber konform verhalten, zwangsläufig mit den geltenden bürgerlichen Regeln und Gesetzen in Konflikt kommen müssen. Damit würde die pädagogisch sehr prekäre Situaion ernsthaft in Erwägung gezogen werden müssen, daß eine subkulturelle Sozialisation in der Unterschicht nahezu zwangsläufig die Gefahr kriminellen Verhaltens - gemessen an den Normen der Mittelschicht - hervorruft.

Eine andere Variante der subkulturtheoretischen Erklärung liegt in der Theorie Albert K. Cohens $(1955,1968)$ vor, der sich darum bemüht hat, bestimmte typische Merkmale der Verhaltensweisen von amerikanischen Großstadtgangs zu erklären. Diese Verhaltensweisen zeichneten sich nach Meinung Cohens dadurch aus, daß sie nicht-utilitaristisch, hedonistisch und bösartig waren. Eine plausible Erklärung für diese drei Merkmale versuchte Cohen dadurch zu erreichen, daß er sie auf eine bestimmte problematische Stellung der Jugendlichen aus unteren sozialen Schichten in der dominanten Mittelschichtkultur zurückführte, die insbesondere durch die Schule repräsentiert wird. In der Schule als klassischer Mittelschichtsinstitution begegnen die Jugendlichen aus unteren, insbesondere der untersten sozialen Schicht einer Lebenswelt, deren Anforderungen sie nur begrenzt gewachsen sind. Sowohl in bezug auf den gesamten Lebensstil, in bezug auf die Kleidung, in bezug auf die sprachliche Kompetenz und in bezug auf die 
Lernvoraussetzungen weisen Jugendliche aus unteren sozialen Schichten erhebliche Handicaps auf, die dazu führen, daß ein erheblicher Teil von ihnen die Situation in der Schule als eine Situation des notorischen Versagens erfährt, die ausgesprochen frustrierend ist. Ein Teil der Jugendlichen aus der unteren sozialen Schicht hält dennoch an dem Ziel des Statuserwerbs innerhalb der "Mittelschichtgesellschaft" - als die er die Gesellschaft durch die „Mittelschichtinstitution Schule kennengelernt hat - fest, obwohl nur wenige Jugendliche dabei erfolgreich sind. Andere Jugendliche geben das Ziel des Aufstiegs in die Mittelschicht auf, arrangieren sich mit der Schule mehr schlecht als recht und sind auf diese Weise in der Lage, ihre potentiell frustrierende Erfahrung relativ gut zu überwinden. Andere Unterschichtjugendliche aber, die am Ziel des gesellschaftlichen Aufstiegs und der Akzeptanz durch die ,,bürgerliche“" Gesellschaft intensivst festhalten, aber ihr Scheitern des Versuchs, dies mit den von der Mittelschichtinstitution Schule vorgesehenen Mittel zu erreichen, nachhaltig erleben, befinden sich in einer Situation des kollektiven Versagens und der kollektiven Frustration, die unter bestimmten Gruppenbedingungen dazu führen kann, daß sie - sich in der Gruppensituation entschieden und entschlossen gegenseitig stimulierend - nun gezielt gegen die Institutionen und die Interessen der Mittelschichtgesellschaft verstoßen . In bestimmten Gruppensituationen agieren sie die massiv angestaute Frustration dadurch aus, daß sie durch bestimmte Verhaltensweisen diese Institutionen zu treffen versuchen. Dies mag beispielsweise dadurch geschehen, daß sie in Kaufhäusern ungezielt bestimmte Gegenstände entwenden, die zwar für sie nicht von materiellem Wert, jedoch von ideellem Wert sind; insofern nämlich als ihre Diebstähle bspw. verdeutlichen, daß sie gegen zentrale Regeln der bürgerlichen Gesellschaft verstoßen. So gesehen ist diese Form der Eigentumskriminalität nicht utilitaristisch, da kein direkter materieller Nutzen daraus resultiert, wohl aber hedonistisch insofern als der Verstoß gegen die Regeln der bürgerlichen Gesellschaft "lusterzeugend" zu sein vermag. In anderen Situationen führt die Frustration der Unterschichtjugendlichen aufgrund der oben genannten Gegebenheiten dazu, daß sie sich ausgesprochen bösartig verhalten, indem sie beispielsweise wehrlosen Opfern schweren körperlichen Schaden zufügen und auf grausame Art und Weise Opfer quälen. Hier spielen bestimmte Gruppenprozesse (vgl. Short/Strodtbeck 1965; Yablonski 1962; 1973) eine zentrale Rolle, die dazu beitragen, daß die empfundenen Frustrationen in sehr ungehemmter Art und Weise in kriminelles Verhalten umgesetzt werden. Für die Bundesrepublik fällt auf, daß man hier diese theoretische Konzeption in bezug auf Kriminalität nicht entscheidend aufgegriffen oder weiterentwickelt hat. Dies mag daran gelegen haben, daß eine so sehr zugespitzte soziale Differenzierung hier lange nicht bestanden hat, die sich jetzt erst durch die Verknüpfung von Arbeitslosigkeit, Armut, ethnischer Diskriminierung etc. zu entwickeln scheint - mit erkennbar negativen Folgeerscheinungen. Dies könnte ein Grund sein, die beispielhafte Studie von Haferkamp (1975) zu replizieren.

\subsubsection{Die Theorie der sozialen Desorganisation}

Verwiesen beide Varianten subkulturtheoretischer Argumentation ganz entschieden auf die Bedeutsamkeit makrosozialer Strukturmerkmale, so gilt dies in abgeschwächter Form auch für bestimmte Varianten der Desorganisationstheorie. Die Slum-Desorga- 
nisationstheorie betont z.B., daf in bestimmten Settings die soziale Situation dadurch gekennzeichnet ist, daß durch ein hohes $\mathrm{Maß}$ an Urbanität und Anonymität, durch eine große Mobilität und durch eine starke Industrialisierung die einstmals vorhandenen sozialen Strukturen zerstört und damit jene Regelsysteme und Kontrollmechanismen, die an sich ein gewisses Ausmaß an Konformität sichern, ,desorganisiert" werden (soziale Desorganisation), so daß die davon betroffenen Individuen in einen Zustand der personalen Desorganisation verfallen, der das Auftreten abweichenden Verhaltens und insbesondere von Kriminalität sehr wahrscheinlich werden läßt (vgl. Cohen 1959). Dies liegt darin begründet, daß für die neu entstandene soziale Situation entweder keine Regelungsmechanismen zur Verfügung stehen oder solche, die miteinander im Widerspruch stehen, so daß keine klare Verhaltensregulierung erfolgen kann. Eine spezielle Variante der Desorganisationstheorie bezieht sich auf die Familie, die durch raschen sozialen Wandel, durch ökonomische Krisen, durch häufige Mobilität, durch räumliche Trennung der Familienmitglieder etc. aus den Fugen geraten kann, so daß sie ihre wichtige sozialisatorische Aufgabe nicht vollständig wahrnehmen kann. Diese theoretische Argumentation, die die sog. Desorganisation der Familie (vgl. allgemein König 1949, $1974 \mathrm{a}, 1974 \mathrm{~b}$ ) in den Vordergrund rückt, hat in der Soziologie abweichenden Verhaltens eine sehr zentrale Rolle gespielt, wobei jedoch das Interesse an dieser Fragestellung eher ideologischen Moden als vorliegenden wissenschaftlichen Erkenntnissen gefolgt ist (vgl. Wilkinson 1974). Die Argumentation hat sich noch dazu häufig auf die Fragestellung der ,unvollständigen Familie“ und ihrer kausalen Bedeutung für Jugendkriminalität eingeengt (vgl. allgemein zu konzeptuellen Ideen und empirischen Befunden Johnson 1986; Wells/Rankin 1986), obwohl vorsichtige Warner (vgl. König 1974 b, S. 114, S. 117) schon lange auf potentielle Denkfehler dieser Argumentation hingewiesen haben. Es wäre dennoch voreilig, wie dies mancher Autor im deutschsprachigen Raum fordert, diese Fragestellung ganz aufzugeben, denn während Albrecht/Howe/Wolterhoff (1991) auf breiter Basis zwar die relativ geringe Relevanz verschiedener Konzeptualisierungen von ,,broken home“ für die selbstberichtete Kriminalitätshäufigkeit zeigen konnten (Ausnahmen: bestimmte Kombinationen von Familienstruktur und sozialer Herkunft sowie bestimmte Altersgruppen (z.B. 16jährige)), berichten immer wieder psychologisch-orientierte Arbeiten (vgl. als hervorragende Übersicht Hetherington 1988) neben bestimmten Reifungsvorsprüngen von Kindern aus unvollständigen Familien von langfristigen psychischen Folgeschäden elterlicher Trennung. Allerdings scheint es auch dabei weniger auf die Unvollständigkeit der Familie an und für sich als vielmehr auf die funktionale Qualität des Familienlebens anzukommen (vgl.eine umfassende Meta-Analyse der deutschen Literatur zum Zusammenhang zwischen Familienvariablen und „psychopathologischen“ Problemen Lösel 1991). Jedenfalls sollte dem Zusammenhang zwischen Familienprozessen und Kriminalität weiter nachgegangen werden, da die Hinweise auf kausale Beziehungen nicht zu übersehen sind (vgl. Wells/Rankin 1991 mit einer neuen Meta-Analyse der internationalen Befunde). 


\subsubsection{Die Theorie der differentiellen Gelegenheiten}

Eine sehr wichtige Integration zweier verschiedener Theorierichtungen stellt die Theorie der differentiellen Gelegenheiten von Cloward und Ohlin (Vgl. Cloward 1968 bzw. Cloward/Ohlin 1960) dar. Diese Theorie bemüht sich darum, einen gravierenden Mangel der Anomietheorie Mertons zu beheben. Während bei Merton eine der zentralen kausalen Variabeln für die Erklärung von Anomie in der Variable ,,Blockierung des Zugangs zu legalen Mitteln zur Erreichung zentraler gesellschaftlicher Werte für bestimmte gesellschaftliche Teilgruppen " bestanden hatte, hatte er sich über die Frage des Zugangs zu bestimmten illegalen bzw. illegitimen Wegen zur Erreichung von gesellschaftlichen Zielen im Grunde nicht geäußert. Zwar vertrat Merton durchaus die Meinung, daß die Akteure auf diese Situation der Blockierung legaler Mittel in verschiedener Art und Weise reagieren können (vgl. verschiedene Reaktionsformen), doch hat er sich zu der sozialen Strukturierung der Wahl dieser Alternativen nicht im einzelnen geäußert. Von Cloward und Ohlin stammt nun die wichtige Einsicht, daß auch die Wahl der illegitimen bzw. illegalen Wege einer sozialen Strukturierung unterliegt, insofern nämlich, als nicht jeder Akteur die gleiche Chance hat, bestimmte illegale Wege zu wählen. Cloward und Ohlin arbeiteten im einzelnen heraus, von welchen sozialstrukturellen und subkulturellen Gegebenheiten es abhängt, welche illegalen Mittel den Akteuren zur Verfügung stehen. Von dieser sozialen und subkulturellen Strukturierung des Zugangs zu illegalen Wegen hängt dann die konkrete Form des gewählten abweichenden Verhaltens $a b$. Unter bestimmten Bedingungen beteiligen sich deviante Jugendliche bei blockierten Chancen im Bereich der legalen Mittel zur Erreichung der zentralen gesellschaftlichen Werte an der Welt des organisierten Verbrechens, unter anderen Bedingungen, in denen ihnen dieser Zugang nicht offen steht, wählen sie möglicherweise die Mitgliedschaft in bestimmten conflict-gangs, die die entstandenen Frustrationen in aggressives Bandenhandeln umsetzen. In wieder anderen sozialstrukturell und subkulturell geprägten Situationen sind den Jugendlichen auch diese beiden Alternativen nicht zugänglich, so daß sie u.U. als sog. ,,doppelte Versager" nur noch den Zugang in die Subkultur der Süchtigen finden. Diese Erweiterung bzw. Synthese aus Anomietheorie und Subkulturtheorie stellt einen gravierenden Fortschritt dar, da mit den genannten Erklärungsmechanismen die verschiedenen Delinquenzphänomene bzw. die altersspezifische, die soziale und ethnische Strukturierung der Kriminalität deutlich besser erklärt werden kann. Insbesondere ist dieser Gedankengang für die geschlechtsspezifische Ausprägung kriminellen Handelns von großer Bedeutung, da sich die spezifische Qualität und Quantität der weiblichen Kriminalität u.a. dadurch erklären läßt, daß die begrenzten Zugangschancen für Frauen auch in den Bereichen des abweichenden Verhaltens mitverantwortlich sind für den Umstand, daß Frauen in bestimmten Bereichen kriminellen Handelns absolut unterrepräsentiert sind (organisiertes Verbrechen, Gewaltkriminalität), während sie in anderen Bereichen im Zuge der Angleichung der Geschlechter schon erheblich an Boden gewonnen haben (s.o.). Ferner würden entsprechende Überlegungen bei einer gewissen Erweiterung auch erklärbar machen, warum sich die bekannten epidemiologischen Befunde in bezug auf die Belastung von Männern und Frauen mit kriminellen Handlungen, mit physischen und psychischen Erkrankungen etc. ergeben. Eine interessante Verknüpfung läßt sich auch zum sog. Routineaktivi- 
täten-Ansatz herstellen, der davon ausgeht, daß Auslösung und spezifische soziale und räumliche Verteilung der Kriminalität eine Funktion der dominanten Routineaktivitäten von Tätern und Opfer darstellen, da sich entsprechende Delikte erst dann ergeben, wenn ein potentieller Täter durch die alltäglichen Aktivitäten einem potentiellen Opfer begegnet, das durch die situativen Gegebenheit keine oder nur geringe Chancen hat, sich oder seinen Besitz vor dem Zugriff zu schützen (vgl. Felson 1986). Merkwürdigerweise hat diese sehr wichtige Theorie, die zudem in ausdrücklich praktisch-politischer Absicht konzipiert worden ist, lange Zeit wenig Beachtung gefunden. Neuerdings belebt sich die Diskussion auch hier, und einige differenzierende Forschungsergebnisse erscheinen (z.B. Simons/Gray 1989; Agnew 1990), die insofern teilweise sehr interessante Ergebnisse liefern als sich zeigt, daß bei Jugendlichen teilweise mit steigenden finanziellen Ressourcen die Delikthäufigkeit steigt - offensichtlich deshalb, weil sie mit diesen Ressourcen einen Lebensstil pflegen können, der die Wahrscheinlichkeit des Zugangs zu kriminellen Handlungen erhöht.

\subsubsection{Die Kontrolltheorie}

Eine weitere soziologische Kriminalitätstheorie läßt sich als späte Folge der psychoanalytischen Kriminalitätstheorien interpretieren, die sich um eine stärkere soziologische Akzentuierung bemüht. Es handelt sich hierbei um die sog. Kontrolltheorie von Hirschi (1969), die sich durch den Umstand yon den meisten anderen Kriminalitätstheorien unterscheidet, daß sie eigentlich nicht das abweichende bzw. kriminelle Verhalten als erklärungsbedürftig ansieht, sondern eher die Konformität. Darin kommt zum Ausdruck, daß diese theoretische Position grundsätzlich davon ausgeht, daß der Mensch ohne besondere soziale Kontrolle und Einbindung im Normalfall abweichendes Verhalten zeigen wird, das auch die Form der Kriminalität annehmen kann. Erst durch bestimmte gesellschaftliche Bindungen und Kontrollmechanismen wird ein Phänomen wie Konformität überhaupt erklärbar. Im einzelnen nimmt Hirschi an, daß verschiedene Arten von Bindungen für die Erzeugung von Konformität verantwortlich sind. Als erstes wäre die Dimension des Attachments zu nennen, die für die emotionalen Bindungen des Menschen an andere Personen, in diesem konkreten Fall für die emotionalen Bindungen von Kindern an ihre Eltern bzw. an ihre Erziehungspersonen, steht. Je intensiver und stärker das Attachment, desto geringer die Wahrscheinlichkeit des Auftretens von kriminellem Handeln. Die Akteure stellen in Rechnung, daß abweichendes Verhalten die emotionale Bindung an die Eltern bzw. an andere Personen gefährden würde, und vermeiden aus diesem Grunde abweichendes Verhalten. Der zweite Typ der Bindung, der bei Hirschi von Bedeutung ist, besteht in dem sog. Commitment, also einer Selbstverpflichtung auf bestimmte Handlungslinien bzw. bestimmte Ziele. Wenn eine Person in bezug auf einen bestimmten Lebensentwurf, z.B. ein bestimmtes berufliches Ziel, Arbeit und Mühe und Verzicht investiert hat, so wird sie das bisher Erreichte vor dem Hintergrund der starken Verpflichtung auf die zentralen Ziele nicht durch abweichendes Verhalten gefährden. Jede Form von kriminellem Handeln könnte die Chance, den eigenen Lebensentwurf entscheidend zu zerstören, deutlich erhöhen. Der dritte Typ der Bindung besteht in einer Verpflichtung auf bestimmte normative Vorstellungen, die im Sozialisationsprozeß erworben werden und von denen man sich im Regelfall nur unter 
ganz spezifischen Bedingungen lösen kann. Je stärker diese normativen Vorstellungen (beliefs) verinnerlicht worden sind, desto unwahrscheinlicher wird es, daß gegen sie ohne Vorliegen weiterer Gründe verstoßen wird, so daß die Beobachtung von Sykes und Matza (1968), daß die weit überwiegende Mehrheit aller Rechtsbrecher im Grunde die gebrochene Norm durchaus akzeptiert und sich wegen des Normbruches bei Entdecktwerden im hohen Maße schämt, plausibel erscheint. Das vierte Band schließlich besteht in einer Form der sozialen Einbindung in bestimmte Netzwerke und Handlungszusammenhänge (involvement), die den Akteuren die Zeit und die Gelegenheit zu kriminellem Handeln nimmt. Dahinter steckt die Alltagsbeobachtung, daß solche Personen, die durch Arbeitsauslastung und durch soziale Kontakte mit geschätzten anderen Personen nahezu nie alleine sind, auch keine Gelegenheit haben, kriminelle Handlungen zu begehen, da sie entweder von vornherein die Verurteilung durch die Anwesenden scheuen oder wegen der Einbindung in bestimmte Aktivitäten gar nicht auf den Gedanken, eine kriminelle Handlung zu begehen, kommen würden. Diese Theorie Hirschis, die in einer Reihe von Punkten modifiziert werden mußte (vgl.u.a. Krohn/Massey 1980), erweist sich in empirischen Untersuchungen als relativ brauchbar (vgl. als Übersicht $L a$ Grange / White 1985; eher negativ dagegen die neueste Überprüfung durch Agnew 1991) und hat den Vorzug, daß sie bestimmte Möglichkeiten der pädagogischen und sozialen Intervention bietet. Andererseits ist sie in einigen Punkten doch entschieden revisionsbedürftig, zumal sie sich in bestimmter Hinsicht ohne Not von einer sozialstrukturellen Betrachtung des Phänomens Kriminalität absetzt. Durch Integration dieses Ansatzes in die allgemeinere Theorie sozialer Netzwerke und des social support dürften sich wichtige Fortschritte für die Kriminalitätstheorie ergeben (zu einem Versuch in diese Richtung vgl. Albrecht u.a.1991).

\subsubsection{Die utilitaristische Theorie der Kriminalität (Abschreckungstheorie)}

Eine weitere neuere Kriminalitätstheorie ist ebenfalls als Neuauflage von eher psychologischen Überlegungen zu verstehen. Es handelt sich dabei um die sog. utilitaristische Theorie kriminellen Handelns, die auch als Abschreckungstheorie bezeichnet wird. Der einfache Gedanke dieser Theorie besteht darin, daß die Häufigkeit kriminellen Handelns sich verstehen läßt als Funktion des Kosten-Nutzen-Kalküls der Akteure (vgl. zu den Grundlagen dieser Theorie u.a. Vanberg 1982; sowie Liska 1982, S. 89 ff.). Während die Nutzenseite des kriminellen Handelns hier zunächst einmal außer acht bleiben kann, hat sich die ganze Aufmerksamkeit aus eher praktisch-politischen Gründen auf die Kostenseite der Kriminalität für den Akteur verlagert. Als relevante Kosten werden insbesondere die Entdeckungswahrscheinlichkeit, die erwartete Sanktionsschwere sowie die Schnelligkeit der Sanktion in Betracht gezogen. Während diese Theorie zunächst allein auf die objektiven Kostenfaktoren zentriert war, hat sich bald ergeben, daß es sinnvoller ist, die subjektiven Wahrnehmungen dieser Kostenfaktoren einzubeziehen. Allerdings zeigte sich alsbald, daß es unhaltbar wäre, von einer additiven Beziehung zwischen diesen Kostenfaktoren und der Wahrscheinlichkeit kriminellen Handelns auszugehen. Dies macht eine simple Überlegung sofort deutlich: Jener potentielle Täter, der die Wahrscheinlichkeit, entdeckt zu werden, für so niedrig hält, daß sie mathematisch gleich null gesetzt werden kann, wird sich auch von einer noch so harten 
Strafe nicht abschrecken lassen, da er ja mit Strafe überhaupt nicht rechnet. Bei Vorliegen einer additiven Beziehung würde sich eine sehr schwere angedrohte Strafe bei einem subjektiven Entdeckungsrisiko von Null in einer mittleren Wahrscheinlichkeit der Begehung einer kriminellen Handlung umsetzen, während - realistischerweise - bei einem multiplikativen Modell eine noch so harte Sanktionsdrohung bei einer subjektiven Entdeckungswahrscheinlichkeit von Null oder annähernd Null ohne abschreckende Wirkung bliebe. Da eine Reihe von vorliegenden empirischen Arbeiten gezeigt hat, daß auf der einen Seite die bisherigen Überprüfungen der Abschreckungstheorie wichtige soziale Kostenfaktoren übersehen haben, insbesondere solche im informellen Bereich (vgl. Meier et al. 1984), während sie andere deutlich überschätzt haben (formale Sanktionierung), läßt sich der wirklich erwartbare Ertrag dieser theoretischen Richtungen nur schwer werten. Offensichtlich berücksichtigt diese Theorie noch zu wenig die Frage des Typs des kriminellen Handelns, um den es geht (mala in se vs. mala prohibita), und beachtet auch allzu wenig die konkreten Umstände, unter denen bestimmte Delikte begangen werden, bzw. die besonderen Merkmale bestimmter Akteursgruppen, die Kosten-Nutzen-Überlegungen in bestimmter Art und Weise strukturieren. In diesem Punkt dürften sich z.B. ältere Kinder und Jugendliche deutlich von jungen Erwachsenen mit eigenen festen Partnerbeziehungen unterscheiden. Zwar vertreten neuerdings nicht wenige Kriminologen die Ansicht, daß dieser Ansatz einen entscheidenden Fortschritt bringen wird (so die meisten Beiträge in Cornish/Clarke 1986), doch kommen die neueren empirischen Studien zu sehr bescheidenen Resultaten (vgl. Bailey/Hubbard 1990; Piliavin et al. 1986; Smith/Gartin 1989; anders dagegen Klepper/Nagin 1989), und Paternoster $(1988,1989)$ meint gar, daß angesichts der Komplexität und der Widersprüchlichkeit seiner Befunde Abhilfe nur durch eine umfassendere Theorie der informellen sozialen Kontrolle gefunden werden kann, die die utilitaristische Theorie in dieser Form nicht bieten kann.

\subsection{Die interaktionistische Theorie abweichenden Verhaltens}

Während alle bisher genannten Theorien sog. ätiologische Theorien waren, die nach bestimmten realen Ursachen für das Auftreten kriminellen Handelns suchen, hat sich ein wesentlicher Fortschritt in der Betrachtung der Kriminalität durch den LabelingAnsatz, eine ganz neue Perspektive, eingestellt, die diese alte Fragestellung als naiv erscheinen läßt. Diese symbolisch-interaktionistische Theorierichtung kommt zu einer Zurückweisung der ätiologischen Fragestellung, da sie den zu erklärenden Sachverhalt, die vermeintliche kriminelle Handlung, erst durch einen nachträglichen Definitionsprozeß, der in Interaktion zustande kommt, als solche konstituiert sieht (vgl. Becker 1963; Sack 1968; Sack 1978). Die vermeintliche kriminelle Handlung trägt nicht bestimmte objektive Verhaltensqualitäten, sondern diese werden erst durch die Beteiligten in einem komplizierten Interaktionsprozeß, bei dem bestimmte Alltagstheorien, Berufsroutinen, aber auch persönliche Interessen, Machtpositionen etc. von Bedeutung sind, diesem Handeln zugeschrieben- oder auch nicht. Da man vor der Handlung selbst gar nicht bestimmen kann, was sie bedeutet, kann man sie auch nicht im üblichen Sinne kausal erklären, denn bei etwas anderem Verlauf des Definitionsprozesses hätte sie vielleicht 
etwas ganz anderes bedeutet. Folgerichtig richtet dieser theoretische Ansatz sein Augenmerk auf ganze andere Fragen: Nicht mehr der Versuch steht im Vordergrund, aus der materiellen oder psycho-sozialen Situation bestimmter Kategorien von Jugendlichen deren kriminelle Handlungen zu erklären, sondern gefragt wird danach, wie es dazu kommt, daß durch bestimmte Reaktionen der Instanzen informeller und formeller sozialer Kontrolle einem prinzipiell sehr verschieden deut- und bewertbarem Handeln in Auseinandersetzung mit den Deutungen und Definitionen der Handelnden und/oder dem Handelnden ein bestimmtes Etikett oder Label zugeschrieben wird - oder auch nicht. Eine solche Position läßt das Hantieren mit offiziellen Statistiken abweichenden Verhaltens sehr fragwürdig erscheinen, da diese Daten durch einen sehr langen und komplexen Prozeß der Realitätskonstruktion entstehen, der von lokalen, regionalen und historischen Randbedingungen - um nur einige Möglichkeiten zu nennen - abhängen kann. Durch diesen Argumentationsgang werden auch fast alle Versuche als relativ wertlos anzusehen sein, durch eine Analyse der offiziellen polizeilichen Kriminalstatistik oder der Gerichtsstatistik zu entscheiden, ob sich im Zeitablauf die Kriminalitätshäufigkeit bei Jugendlichen erhöht hat etc. Erst wenn man gezeigt hat, daß sich im betreffenden Zeitraum weder die Anzeigebereitschaft der Bevölkerung, noch die Ermittlungsbereitschaft und Sanktionsbereitschaft von Polizei und Staatsanwaltschaft bzw. Gericht verändert haben, kann man won steigenden Häufigkeitsziffern auf eine wirkliche Zunahme ,krimineller“ Handlungen schließen. Tatsächlich kann man sehr glaubhaft machen, daß ein erheblicher Teil der z.B. für die 70 er Jahre oft behaupteten kräftigen Zunahme der Jugendkriminalität daraus resultiert, daß - bedingt durch zunehmende Anonymität der Lebenszusammenhänge, durch reduzierte Toleranz, durch (oft unberechtigtes) Vertrauen auf die Handlunskompetenz professioneller Sozialkontrolleure etc. - gegenüber früher eine deutlich verstärkte Neigung besteht, wahrgenommenes abweichendes Verhalten zum AnlaB zu nehmen, formale Kontrollinstanzen zu informieren oder zu mobilisieren und auf diese Weise auch früher immer schon aufgetretene Ungezogenheiten, Streiche und übliche Händel zu kriminellen Handlungen zu machen (vgl. Albrecht/Lamnek 1979).

Eine ganz wesentliche Erkenntnis dieses Ansatzes besteht auch und gerade darin, aufgezeigt zu haben, daß Form und Inhalt dieser Definitionsprozesse eine Art Eigendynamik aufweisen, die es erforderlich erscheinen lassen, diese ,Kreation“ abweichenden Verhaltens durch ,gesellschaftliche Reaktion“" im Längsschnitt zu untersuchen (vgl. Albrecht / Karstedt-Henke 1987). Insbesondere betont dieser Ansatz, daß durch die spezifische Ausprägung dieses Definitionsprozesses der ,abweichende Akteur" in doppelter Weise betroffen zu sein pflegt, denn erstens gehen mit den informellen, vor allem aber mit den formellen Sanktionen und deren oft unbeabsichtigten Nebenfolgen Beschneidungen des Rollenrepertoires einher (Role engulfment bei Becker 1963), die von ganz erheblicher langfristiger und irreversibler Bedeutung sein können (Berufsverbot für bestimmte Berufe, Versperrung des Studiums etc.), da sie die gesamte weitere Laufbahn betreffen können. Zweitens können derartige Definitionsprozesse, auch wenn sie rechtlich gesehen u.U. ohne gravierende Folgen bleiben, auf den betroffenen Akteur in fataler Weise einwirken, indem sie unter bestimmten Umständen Identitätstransformationen oder zumindest Selbstkonzept- bzw. Selbstwertveränderungen auslösen (vgl. als Übersicht Albrecht/van Kampen 1991). Hierbei ist allerdings zu unterscheiden, ob die Reaktionen bspw. im informellen Bereich durch solche Personen erfolgen, die das deviante 
Handeln möglicherweise positiv bewerten (z. B. peers von delinquenten Jugendlichen), oder aber durch formelle Instanzen sozialer Kontrolle, die ihre Reaktion als eindrucksvolle öffentliche „Degradationszeremonie“" inszenieren, durch die der Akteur so massiv stigmatisiert wird, daß es zu einer retrospektiven Neuinterpretation der gesamten Biographie kommt, die ihn als ,zutiefst anders“, als Außenseiter diskreditiert. Dadurch wiederum - so der besonders provokative Gedanke dieser Theorie - kommt es in der Regel erst zu dem, was die gesellschaftliche Reaktion schon unterstellt: War die erste abweichende Handlung, deren abweichende Qualität ohnehin erst post hoc zustande kommt, verstehbar als Resultat eines reinen Zufallsprozesses (primäre Devianz; vgl. Lemert 1967, 1975), so handelt der als deviant Etikettierte und Stigmatisierte in Reaktion auf diesen Degradationsprozeß, der zu einer Identitätsstransformation geführt hat, mit erhöhter Wahrscheinlichkeit deviant (sekundäre Devianz). Dabei gibt es gute Gründe dafür anzunehmen, daß gesellschaftliche Reaktionen dieser Art bei Personen, deren Identitätsbildungsprozeß aufgrund der typischen Verlaufsmuster der Biographieentwicklung in einer kritischen Phase ist, wie dies bei der Jugend der Fall ist, besonders gravierende Auswirkungen haben, während sie bei Personen mit konsolidierter Identität vergleichsweise geringe Folgen haben werden (vgl. Heiss 1981). Damit ergibt sich eine ganz eigentümliche Beziehung zwischen abweichendem Verhalten und Jugend: Man kann aus $z$ wingenden Gründen annehmen, daß die Jugend als eine brisante Übergangsphase, in der eine aus bestimmten Gründen auftretende Kumulation von Statuspassageproblemen in einem solchen Ausmaß zu Streß fuihrt, daß neben einigen anderen, teilweise problematischen Coping-Strategien (wie z.B. Drogenkonsum) auch zu kriminellen Handlungen gegriffen wird, auch jene Phase ist, in der gesellschaftliche Etikettierungen besonders explosive Folgen haben. Aus diesem Grunde ist diese Phase für die Problematik der kriminellen Karriere von zentraler Bedeutung und die Qualität der informellen und formellen sozialen Kontrolle so entscheidend wichtig. Angesichts dieser Tatsache ist es sehr erstaunlich, daß es nicht sehr viele Studien gibt, die die Auswirkungen formaler und informeller Etikettierungen auf methodisch einwandfreie Weise nachzuprüfen versucht haben. Es zeigt sich allerdings auch, daß man diese Effekte nicht überschätzen sollte (vgl. Übersicht bei Albrecht u.a. 1991).

\subsection{Neuere integrative Ansätze}

Dieser zuletzt vorgetragene Gedanke erhält seine ganze Tragweite, wenn man eine relativ neue sozialpsychologische Theorie der Abweichung hinzuzieht, die abweichendes Verhalten u.a. als einen Versuch der Verteidigung des Selbstwertes deutet (Kaplan 1975; 1980). Diese Theorie nimmt - äußerst verkürzt dargestellt - an, daß der Mensch ein Bedürfnis nach Erhaltung bzw. Steigerung seines Selbstwertes hat, so daß er dann, wenn er aufgrund mangelnder Fähigkeit, den Standards seiner Bezugsgruppe zu entsprechen, vor das Problem der Unterschreitung eines bestimmten Minimums an Selbstachtung gestellt wird, durch die Begehung von devianten Handlungen, die in den Augen einer neuen, devianten Bezusgruppe Zustimmung und Anerkennung finden, eine Steigerung seines Selbstwertes erfährt, wodurch die Gefahr weiterer Straftaten steigt, da sie ja Achtung bringen, wenn auch nur in den Augen der neuen Bezugsgruppe. Das aber 
heißt, daß unangemessene Reaktionen auf Leistungsversagen und auf Verhaltensprobleme den Jugendlichen in eine Situation bringen können, aus der ihm nur die Begehung von abweichenden, oft eben auch kriminellen Handlungen herauszuhelfen scheint. Demnach wäre nicht in jedem Falle die Folge kriminellen Handelns ein schlechtes Selbstkonzept, sondern häufig eher die Ursache; ja kriminelles Handeln kann nach Kaplan unter bestimmten Bedingungen zu einer Selbstwertsteigerung führen; und wenn die Lerntheorie stimmte, dann würde sich daraus die Gefahr weiterer Delikte eindeutig ergeben. Nun ist die empirische Forschung zu dieser Frage jedoch bisher zu keinem eindeutigen Ergebnis gekommen (vgl. z.B. Albrecht/van Kampen 1991; Rosenberg/Schoenbach/Schooler 1989; Scheff/Retzinger/Ryan 1989; Wells 1989), so daß überzogene Schlußfolgerungen vermieden werden sollten. Es spricht aber vieles dafür, daß Selbstwertsteigerungen durch Kriminalität nur bei solchen Jugendlichen auftreten, die zunächst einen äußerst niedrigen Selbstwert aufweisen. Ferner scheint es so zu sein, daß die relative Bedeutung des Selbstwertes - verglichen mit anderen kausalen Variablen - bei Mittelschichtjugendlichen größer ist als bei Unterschichtjugendlichen. Wie dem auch immer sei, während die klassische interaktionistische Position für viele Fälle jugendlicher Kriminalität die Strategie der ,radikalen Nicht-Intervention“ empfahl (Schur 1973) und dafür viele Gründe angeben konnte, melden sich nun Autoren zu Wort, die die diesem Modell unterliegende "Normalisierungsstrategie“" aufgrund theoretischer Überlegungen bedenklich finden. Sie empfehlen statt dessen, die aufgetretenen Fälle jugendlicher Devianz durchaus mißbilligend und kritisierend zur Kenntnis zu nehmen, ja sogar den betreffenden Akteur zu ,,beschämen“, aber in einer solchen Art und Weise, daß er sich dennoch nicht als ausgeschlossen fühlen muß, da die Kritik und Sanktionierung ihm deutlich machen können, daß man ihm eine Chance gibt. Durch dieses ,reintegrative shaming“ könnten und sollten die moralischen Grenzen der Gemeinschaft verdeutlicht, aber Stigmatisierungen, die durch neue Subkulturbildungen neue Kriminalitätsursachen hervorrufen würden, vermieden werden (vgl. Braithwaite 1989). So interessant dieser Ansatz zu sein scheint, so bleibt er doch in bezug auf die Vorstellungen für die Entwicklung solcher Sanktionsprozeduren zu vage und beschwört andererseits die Gefahr herauf, daß jene, die immer schon für eine etwas ,entschiedenere Gangart" waren, sich irrtümlicherweise bestätigt sehen.

\section{Ausblick in theoretischer und praktischer Hinsicht}

\subsection{Theoretische Entwicklungen}

Versucht man eine Bilanz der theoretischen Ansätze zur Erklärung von Jugendkriminalität, so fällt auf, daß die Mehrheit der existierenden Theorien zwar in der Auseinandersetzung mit empirischen Daten formuliert worden sind, die an jugendlichen Populationen gewonnen worden sind, daß sie aber dennoch die besonderen Probleme, die die Jugendphase bestimmen und die für die Stellung der Jugendlichen in modernen Gesellschaften bezeichnend sind, nicht systematisch für die Theoriebildung beachten. Wenn 
sie dies dennoch tun, so wählen sie aus den vielen wichtigen Aspekten der Jugendproblematik einige isolierte heraus, so daB meist nicht einmal Theorien mittlerer Reichweite resultieren. Nichtsdestoweniger sollte deutlich geworden sein, daB wir über einen bunten Strauß sehr unterschiedlicher Theorieansätze verfügen, die zumindest für die Erklärung von Teilaspekten relevant sein dürften und die für die gesellschaftliche Praxis des Umgangs mit der Kriminalität von Jugendlichen wichtige Anregungen geben könnten. Eine der ganz wesentlichen Voraussetzungen für entschiedene Fortschritte in der Theorie der Jugendkriminalität wäre jedoch, daß die Kriminalitätstheorien sich auf der einen Seite um eine systematische Berücksichtigung der sehr fruchtbaren theoretischen Entwicklungen in der allgemeinen Jugendforschung bemühen und auf der anderen Seite zur Kenntnis nehmen, daß die Kriminalität nur eine der verschiedenen möglichen problematischen Reaktionsweisen von Akteuren auf belastende Ereignisse und Situationen darstellt (z.B. neben psychischen Erkrankungen, physischen Erkrankungen, Drogenabhängigkeit, Suizid etc.), deren Auswahl durch den jeweiligen Akteur erklärt werden muß, wenn man einen wirklichen Erkenntnisfortschritt erreichen will.

\subsection{Entwicklungstrends in der Kontrollpolitik}

Ohne Zweifel lehrt ein Blick auf die Entwicklung des Umgangs mit der Jugendkriminalität, daß die Fortschritte in diesem Bereich bescheiden sind - jedenfalls verglichen mit dem, was sich Sozialwissenschaftler vorstellen könnten (vgl. Albrecht 1982). Dabei darf aber nicht übersehen werden, daß die Sozialwissenschaftler selbst durch etwas voreilige Slogans wie „Nothing works“ (vgl. vor allem Lipton/Martinson/Wilks 1975) die Basis für die gesellschaftliche Abstinenz von Neuerungen mitgelegt haben. Diese Meinung war keinesfalls leichtfertig in die Welt gesetzt worden, sondern Ergebnis sehr ernsthafter Systematisierungsversuche der Evaluationsforschungen zur Behandlung bzw. der Intervention im Bereich der Kriminalität, die zur wissenschaftlichen Begleitung der vielen neuen Modelle durchgeführt worden waren, und noch heute tobt ein erbitterter Streit darüber, ob für die neueren Modelle nicht doch das gleiche negative Ergebnis gilt (vgl. bejahend dazu Whitehead/Lab 1989, entschieden in die gegenteilige Richtung dagegen Andrews et al. 1990). Die dabei gewählten Behandlungsmethoden und Modelle sind Legion und können hier nicht dargestellt werden.

Blicken wir nur auf einige wenige Modelle im Bereich der Jugendkriminalität, so schienen insbesondere jene Modelle vielversprechend, die sich um eine frühzeitige Herauslenkung (Diversion) delinquenter Jugendlicher aus dem Kriminaljustizsystem bemühen, die nach den Regeln des \$ 45 JGG in verschiedenen Formen, die hier nicht dargestellt werden können, immer schon möglich, ja vom Gesetzgeber offensichtlich gewollt, von den Staatsanwaltschaften jedoch relativ selten genutzt worden waren. In den letzten Jahren hat sich diese Praxis - wenn auch regional und lokal sehr unterschiedlich - deutlich ausgeweitet und nachweislich empirisch bewährt (Heinz/Hügel 1986/87). Gleiches gilt für die richterliche Diversion nach § $47 \mathrm{JGG}$, die aber insofern nur eine nachrangige Bedeutung haben sollte, als sie im Grunde schon durch den Staatsanwalt hätte praktiziert werden können.

Gegen viele dieser neuen Praktiken gibt es erhebliche Einwände. Während die eher konservative Position befürchtet, daß der Verzicht auf formelle Verfahren und insbeson- 
dere der Verzicht auf solche Maßnahmen, die zumindest subjektiv Bestrafungscharakter haben, bei den Betreffenden, vor allem aber bei den Jugendlichen allgemein, zu einer Normerosion führt, die mittel- und langfristig die Kriminalität wird ansteigen lassen, fürchten verschiedene kritisch-fortschrittliche Geister zum einen, daß die Informalisierung zu einem erheblichen Verlust an Rechtsstaatlichkeit führen kann, der sich insbesondere durch Rechtsungleichheit und Rechtsunsicherheit umschreiben ließe, zum anderen, daß die Vertreter der pädagogischen und sozialarbeiterischen Berufe, aber auch die Polizei und die Staatsanwaltschaften wegen der Annahme, eine Interventionsmaßnahme gegen bestimmte deviante Jugendliche ende ohnehin nahezu nie in einer strafrechtlich relevanten Entscheidung, verstärkt zu offiziellen Kontroll- und Interventionsmaßnahmen greifen würden, um dem Jugendlichen zu ,helfen“, und zwar auch bei Verhaltensweisen und Problemkonstellationen, in denen man bisher - mit Recht eher weggesehen oder nur mit dem Finger gedroht habe (net widening).

Die bisher vorliegenden Forschungsergebnisse haben für die Bundesrepublik keine eindeutigen Hinweise auf solche negativen Begleiterscheinungen erbracht, wohl aber gezeigt, daß die verschiedenen neuen Modelle wie Täter-Opfer-Ausgleich, Konfliktschlichtungsgespräche, Gruppenpädagogik, Erlebnispädagogik, Wiedergutmachung, gemeinmützige Arbeiten etc. mindestens genau so gute Ergebnisse, meist jedoch bessere Ergebnisse erzielen als die alte Praxis. Noch sind wir aber noch weit davon entfernt, das vorhandene theoretische und empirische Wissen phantasievoll in einen wirklich neuen Umgang mit der Jugendkriminalität umgesetzt zu haben.

\section{Literatur}

Adler, F.: Sisters in Crime: The Rise of the New Female Criminal. New York 1975

Adler, F.: The Incidence of Female Criminality in the Contemporary World. New York 1981

Agnew, R.: Adolescents Resources and Delinquency. In: Criminology 28 (1990), S. 535-565

Agnew, R.: A Longitudinal Test of Social Control Theory. In: Journal of Research in Crime and Delinquency 28 (1991), S.126-156

Akers, R.L. / Krohn, M.D. / Lanza-Kaduce, L. / Radosevich, M.: Social Learning and Deviant Behavior: A Specific Test of a General Theory. In: American Sociological Review 44 (1979), S. 636-655

Akers, R.L.: Deviant Behavior: A Social Learning Approach, 3. Aufl. Belmont, C.A., 1985

Albrecht, G.: Zwerge auf den Schultern eines Riesen? Neuere Beiträge der Theorien abweichenden Verhaltens und sozialer Kontrolle in der Tradition Emile Durkheims. In: Alemann, H. von/Thurn, H.P. (Hrsg.): Soziologie in weltbürgerlicher Absicht. Festschrift für René König zum 75. Geburtstag. Opladen 1981, S.323-358

Albrecht, G.: Muß angewandte Soziologie konforme Soziologie sein? Zum Verhältnis von Theorie und angewandter Soziologie im Bereich des abweichenden Verhaltens und der sozialen Kontrolle. In: Beck, U. (Hrsg.): Soziologie und Praxis, Göttingen 1982, S. 161-204

Albrecht, G.: Möglichkeiten und Grenzen der Prognose „Krimineller Karrieren“" In: Deutsche Vereinigung für Jugendgerichte und Jugendgerichtshilfen (Hrsg.): Mehrfach Auffällige- Mehrfach Betroffene. Erlebnisweisen und Reaktionsformen. Bonn 1990, S. 99-116

Albrecht, G. / Karstedt-Henke, S.: Alternative Methods of Conflict-Settling and Sanctioning: Their Impact on Young Offenders. In: Hurrelmann, K. / Kaufmann, F.-X./Lösel, Fr. (Hrsg.): Social Intervention: Potential and Constraints. Berlin-New York 1987, S.315-332

Albrecht, G. / et al.: Alternative Konfliktlösungs- und Sanktionierungspraktiken: Auswirkungen auf strafrechtlich Auffälige. Finanzierungsantrag des SFB 227 für die dritte Förderungsphase
$(1992-1994)$, Bielefeld 1991 
Albrecht, G. / Howe, C.-W. / Wolterhoff, J.: Familienstruktur und Delinquenz. In: Soziale Probleme 2 (1991). S. $107-156$

Albrecht, G. / Howe, C.-W. / Wolterhoff-Neetix, J.: Neue Ergebnisse zum Dunkelfeld der Jugenddelinquenz: Selbstberichtete Delinquenz von Jugendlichen in zwei westdeutschen Großstädten. In: Kaiser, G. / Kury, H. / Albrecht, H.J. (Hrsg.): Kriminologische Forschungen in den 80er Jahren. Projektberichte aus der Bundesrepublik Deutschland. 2. Halbbd. Freiburg 1988, S. $661-696$

Albrecht, G. / van Kampen, N.: Auswirkungen der Diversion auf die Entwicklung des Selbstbildes delinquenter Jugendlicher. Universität Bielefeld. SFB 227, Preprint Nr. 45, 1991

Albrecht, G. / Howe, C. W.: Soziale Schicht und Delinquenz. Verwischte Spuren oder falsche Fährte?, Universität Bielefeld, SFB 227, Preprint Nr. 43, 1991 (erscheint in gekürzter Form in der Kölner Zeitschrift für Soziologie und Sozialpsychologie, 1992)

Albrecht, H.-J.: Die sanfte Minderheit. In: Bewährungshil fe 34, 1987, S. 341 - 359

Albrecht, P.A. / Lamnek, S. Jugendkriminalität im Zerrbild der Statistik. Eine Analyse von Daten und Entwicklungen. München 1979

Albrecht, P. A. / Lamott, F.: Innenansichten. Gruppendiskussionen mit Bediensteten des Jugendstrafvollzugs. In: Albrecht, P.A. / Schüler-Springorum, H. (Hrsg.): Jugendstrafe an Vierzehn-und Fünfzehnjährigen. Strukturen und Probleme. München 1983, S.135-176

Amelang, M.: Sozial abweichendes Verhalten. Entstehung, Verbreitung, Verhinderung. Berlin 1986

Andrews, D. A. / et al. : Does Correctional Treatment Work? A Clinically Relevant and Psychologically Informed Meta Analysis. In: Criminology 28 (1990), S. 369-404

Ariès, P.: Geschichte der Kindheit. München 1975

Bachman, J.G.: Die Bedeutung des Bildungsniveaus für Selbstwertgefühl, berufsbezogene Einstellungen, Delinquenz und Drogenkonsum von Jugendlichen. In: Olbrich, E. /Todt, E. (Hrsg.): Probleme des Jugendalters. Neuere Sichtweisen. Berlin etc. 1984, S. $131-157$

Bailey, S.L. / Hubbard, R.L.: Developmental Variation in the Context of Marijuana Initiation among Adolescents. In: The Journal of Health and Social Behavior 31 (1990), S. 58 - 70

Becker, H.S.: Outsiders: Studies in the Sociology of Deviance. New York 1963

Bernard, T.: Control Criticisms of Strain Theories. An Assessment of Theoretical and Empirical Adequacy. In: Journal of Research in Crime and Delinquency 21 (1984), S. 353 - 372

Bernard, T.: Testing Structural Strain Theories. In: Journal of Research in Crime and Delinquency 24 (1987), S. $262-280$

Bielefeld, U.: Exkurs: Geschlossene Heime als Alternative zum Jugendstrafvollzug? Eine Auswertung von Literatur zur Heimunterbringung. In: Albrecht, P. A. / Schüler-Springorum, H. (Hrsg.): Jugendstrafe an Vierzehn- und Fünfzehnjährigen. Strukturen und Probleme. München 1983, S. $177-185$

Bohle, H.H.: Soziale Abweichung: Die Anomietheorie in der Diskussion. Darmstadt-Neuwied 1975 Boor, C. de: Soziotherapie als angewandte Psychoanalyse in einer Sondereinrichtung der holländischen Justiz. In: Lüderssen, K. / Sack, F. (Hrsg.): Seminar: Abweichendes Verhalten III. Frankfurt a.M. 1977 , S. $402-416$

Braithwaite, J.: Crime, Shame and Reintegration. Cambridge etc. 1989

Brusten, M. / Hurrelmann, K.: Abweichendes Verhalten in der Schule. Eine Untersuchung zu Prozessen der Stigmatisierung. München 1973

Burgess, R.L./Akers, R.L.: A Differential Association-Reinforcement Theory of Criminal Behavior. In: Social Problems 14 (1966), S. $128-147$

Cernkovich, St. A./Giordano, P.C.: Family Relationships and Delinquency. In: Criminology 25 (1987), S. $295-322$

Cloward, R.A. /Ohlin, L.E.: Delinquency and Opportunity. A Theory of Delinquent Gangs. New York 1960

Cloward, R.A.: Illegitime Mittel, Anomie und abweichendes Verhalten. In: Sack, F./König, R. (Hrsg.): Kriminalsoziologie. Frankfurt a.M. 1968, S. 314-338

Cohen, A. K.: Zur Erforschung delinquenter Subkulturen. In: Sack, F. / König, R. (Hrsg.): Kriminalsoziologie. Frankfurt a.M. 1968, S. $372-394$

Cohen, A.K.: Kriminelle Jugend. Hamburg 1955 
Cohen, A.K.: The Study of Social Disorganization and Deviant Behavior. In: Menton, R.K. / Brown. L. / Cottrell, L. (Hrsg.): Sociology Today. Problems and Prospects. New York 1959, S. $461-484$ Cohn, E.S. / White, S.O.: Legal Socialization. A Study of Norms and Rules. New York etc. 1990

Cornish, D.B. / Clarke, R.V. (Hrsg.): The Reasoning Criminal-Rational Choice Perspectives on Offending. New York-Berlin etc. 1986

Durkheim, E.: Die Regeln der soziologischen Methode. Neuwied 1961

Durkheim, E.: Über die Teilung der sozialen Arbeit. Frankfurt a.M. 1977 (2. Aufl. unter dem Titel "Über soziale Arbeitsteilung". Frankfurt a.M. 1988)

Felson, M.: Linking Criminal Choices, Routine Activities, and Informal Control and Criminal Outcomes. In: Cornish, D.B. / Clarke, R.V. (Hrsg.): The Reasoning Criminal. New York-Berlin etc. 1986

Gephart, W.: Strafe und Verbrechen. Die Theorie Emile Durkheims. Opladen 1990

Haferkamp, H.: Kriminelle Karrieren. Handlunsgtheorie, Teilnehmende Beobachtung und Soziologie krimineller Prozesse. Reinbek 1975

Hagan, J./Gillis, A.R./Simpson, J.: The Class Structure of Gender and Delinquency: Toward a Power-Control Theory of Common Delinquent Behavior. In: American Journal of Sociology 90 (1985), S. $151-178$

Hagan, J. / Gillis, A.R. / Simpson, J.: Class in the Household: A Power-Control Theory of Gender and Delinquency. In: American Journal of Sociology 92 (1987), S. $788-816$

Heinz, W./Hügel, Chr.: Erzieherische Maßnahmen im deutschen Jugendstrafrecht. Bonn 1986

Heiss, J.: The Social Psychology of Interaction. Englewood Cliffs, N.J., 1981

Hetherington, E.M. / Anderson, E.R.: The Effects of Divorce and Remarriage on Early Adolescents and Their Families. In: Levine, M.D. / McAnarney, E.R. (Hrsg.): Early Adolescent Transitions. Lexington und Toronto 1988, S. 49-67

Hirschi, Tr.: Causes of Delinquency. Berkeley 1969

Irvine, R.: Legal Socialisation- A Critique of a New Approach. In: Farrington, P./Hawkins, K. /Lloyd-Bostock, S.M. (Hrsg.): Psychology, Law and Legal Process. London 1979

Johnson, R.E.: Family Structure and Delinquency: General Patterns and Gender Differences. In: Criminology 24 (1986), S. 65 - 80

Kaiser, G.: Jugendstrafrecht. In: Kaiser, G. / Kerner, H.-J./Sack, F. / Schellhoss, H. (Hrsg.): Kleines Kriminologisches Wörterbuch. 2. neubearb. Aufl. Heidelberg 1985, S. $172-177$

Kaplan, H.B.: Deviant Behavior in Defense of Self. New York 1980

Kaplan, H.B.: Self-Attitudes and Deviant Behavior. Pacific Palisades 1975

Kerner, H.-J.: Verbrechenswirklichkeit und Strafverfolgung. München 1972

Kersten, J. / Kreissl, R. / von Wolffersdorff-Ehlert, Chr.: Die sozialisatorische Wirkung totaler Institutionen. Eine Analyse von Lebensbildern. In: Albrecht, P.A./Schüler-Springorum, H. (Hrsg.): Jugendstrafe an Vierzehn- und Fünfzehnjährigen. Strukturen und Probleme. München 1983, S.

Klepper, St. / Nagin, D.: The Deterrent Effect of Perceived Certainty and Severity of Punishment Revisited. In: Criminolgy 27 (1989), S. $721-746$

Kohlberg, L.: From Is to Ought: How to Commit the Naturalistic Fallacy and Get Away with It in the Study of Moral Development. In: Mischel, T. (Hrsg.): Cognitive Development and Epistemology.
New York 1971, S. 151-235 Kohlberg, L.: Essays on Moral Development, I.: In the Philosophy of Moral Development, New York
1981 Kohlberg, L.: Moral Stages and Moralization. In: Likona, T. (Hrsg.): Moral Development and Beha-
vior. New York 1976, S. 31 -53

Kohlberg, L.: Stage and Sequence: The Cognitive-Developmental Approach to Socialization. In: Goslin, D.A. (Hrsg.): Handbook of Socialization Theory. Chicago 1969, S. $347-480$

Kohlberg, L.: The Psychology of Moral Development: The Nature and Validity of Moral Stages. San
Francisco 1984

König, R.: Das Recht im Zusammenhang der sozialen Normensysteme. In: Hirsch, E.E./ Rehbinder,
M. (Hrsg.): Studien und M. (Hrsg.): Studien und Materialien zur Rechtssoziologie. Sonderheft 11 der Kölner Zeitschrift für
Soziologie und Sozialpsychologie. Köln-Opladen 1976, S. 36-53 
König, R.: Die Familie in der Gegenwart. München $1974 \mathrm{~b}$

König, R.: Materialien zur Soziologie der Familie, Neuaufl. Köln 1974a

König, R.: Soziologie der Familie. In: König, R. (Hrsg.): Handbuch der empirischen Sozialforschung, 2., revid. Aufl., Bd. 7. Stuttgart 1976, S. $1-217$

König. R.: Überorganisation der Familie als Gefährdung der seelischen Gesundheit. In: PfisterAmmende, M. (Hrsg.): Die Psychohygiene. Grundlagen und Ziele. Bern 1949, S. 130-144

Krohn, M.D. / Massey, J.L. : Social Control and Delinquent Behavior. An Examination of the Elements of the Social Bond. In: Sociological Quarterly 21 (1980), S. 529-543

Lab, St. P. / Whitehead, J.T : From „Nothing Works" to „The Appropriate Works": The Latent Stop on the Search for the Secular Grail. In: Criminology 28 (1990), S.405-417

LaGrange. R.L./ White, H. R. : Age Differences in Delinquency: A Test of Theory. In: Criminology 23 (1985), S. $19-45$

Lamnek, S.: Sozialisation und kriminelle Karriere. In: Schüler-Springorum, H. (Hrsg.): Mehrfach auffälig. München 1982, S. 13-85

Lamnek, S.: Theorien abweichenden Verhaltens, 2. Aufl., München 1983

Lemert, E.M.: Der Begriff der sekundären Devianz. In: Lüderssen, K. / Sack, F. (Hrsg.): Seminar Abweichendes Verhalten I. Die selektiven Normen der Gesellschaft. Frankfurt a. M. 1975, S. 433-476

Lemert, E.M.: Human Deviance, Social Problems and Social Control. Englewood Cliffs, N.J., 1967

Liska, A. E.: Perspectives on Deviance. Englewood Cliffs, N.J., 1981

Lösel, Fr. (Hrsg.): Kriminalpsychologie. Weinheim 1983

Lösel, Fr.: Handlungskontrolle und Jugenddelinquenz. Stuttgart 1975

Lösel, Fr.: Meta-Analysis and Social Prevention: Evaluation and a Study on the Family-Hypothesis in Developmental Psychopathology. In: Albrecht, G. /Otto, H.-U. (Hrsg.): Social Prevention and the Social Sciences. Theoretical Controversies, Research Problems, and Evaluation Strategies. BerlinNew York 1991, S.305 - 332

Matza, D.: Delinquency and Drift. New York 1967

McCord, J.: Crime in Moral and Social Contexts. In: Criminology 28 (1990), S.1-26

Mead, G.H.: Geist, Identität und Gesellschaft. Frankfurt a.M. 1968 (zuerst Chicago 1934)

Meier, R. F. / Burkett, St. R./Hickman, C. A.: Sanctions, Peers, and Deviance. Preliminary Models of Social Control Process. In: Sociological Quarterly 25 (1984), S.67-82

Merton, R.K.: Anomie, Anomia, and Social Interaction: Contexts of Deviant Behavior. In: Clinard, M.B. (Hrsg.): Anomie and Deviant Behavior. A Discussion and Critique. New York 1964, S. 213-242

Merton, R.K.: Social Theory and Social Structure, 2., rev. Aufl. New York 1957 (zuerst Glencoe, Ill. 1951)

Miller, W. B.: Die Kultur der Unterschicht als ein Entstehungsmilieu für Bandendelinquenz. In: Sack, F. / König, R. (Hrsg.): Kriminalsoziologie. Frankfurt a.M. 1968, S. 339 - 359 (zuerst 1958)

Nye, F.I. / Short, J.F. /Olson, V.J.: Socio-Economic Status and Delinquent Behavior. In: American Journal of Sociology 63 (1958), S. $381-389$

Offer, D.: Das Selbstbild normaler Jugendlicher. In: Olbrich, E. / Todt, E. (Hrsg.): Probleme des Jugendalters. Neuere Sichtweisen. Berlin etc. 1984, S. 111 - 130

Olbrich, E.: Jugendalter- Zeit der Krise oder der produktiven Anpassung?. In: Olbrich, E./ Todt, E. (Hrsg.): Probleme des Jugendalters. Neuere Sichtweisen. Berlin etc. 1984, S.1-46

Opp, K.-D.: Abweichendes Verhalten und Gesellschaftsstruktur. Darmstadt und Neuwied 1974

Parsons, T.: The Social System. New York 1951

Parsons, T.: Entstehung und Richtung abweichenden Verhaltens. In: Sack, F. / König, R. (Hrsg.): Kriminalsoziologie. Frankfurt a.M. 1968, S. 249-260

Paternoster, R.: Examining Three-Wave Deterrence Models: A Question of Temporal Order and Specification. In: The Journal of Criminal Law and Criminology 79 (1988), S. 135 - 178

Paternoster, R.: Decisions to Participate in and Desist from Four Types of Common Delinquency: Deterrence and the Rational Choice Perspective. In: Law and Society Review 23 (1989), S. 7-40

Peters, H.: Kriminalität und Familie. In: Nave-Herz, R./Markefka, M. (Hrsg.): Handbuch der Familien- und Jugendforschung, Bd. 1. Neuwied-Frankfurt a.M. 1989, S. 577-593 
Platt, A.M.: The Child Savers: The Invention of Delinquency. Chicago 1969

Redl, F. / Wineman, D.: Children Who Hate. New York [951

Rosenberg, M. / Schoenbach, C. / Schoolet, C.: Self-Esteem and Adolescent Problems: Madelling Reciprocal Effects. In: American Sociological Review 54 (1989), S.1004- 1018

Sack, F.: Neue Perspektiven in der Kriminalsoziologie. In: Sack. F. / König. R. (Hrsg.): Kriminalsoziologie. Frankfurt a.M. 1968, S. 400-431

Sack, F.: Probleme der Kriminalsoziologie. In: König, R. (Hrsg.): Handbuch der empirischen Sozialforschung, 2, revid. Auflage Stuttgan 1978, S. $192-492$

Schaffstein, E.: Jugendstrafrecht. Stutigart 1983

Schumann, K.F. / Berlitz, C. / Guth, H.-W. / Kaulitzki, R. : Jugendkriminalität und die Grenzen der Generalprävention. Eine empirische Untersuchung. Bremen 1985

Schur, E. M.: Abweichendes Verhalten und Soziale Kontrolle. Etikettierung und gesellschafiliche Reaktionen. Frankfurt a.M. 1974

Schur, E. M.: Interpreting Deviance. A Sociological Introduction. New York etc. 1979 Schur, E.M.: Radical Nonintervention: Rethinking the Delinquency Problem. Englewood Cliffs, N.J..
1973

Seiffge-Krenke, I.: Formen der Problembewältigung bei besonders belasteten Jugendlichen. In: OIbrich, E. / Todt, E. (Hrsg.): Probleme des Jugendalters. Berlin etc. 1984, S. 353-386

Short, J.F. / Nye, F.I.: Erfragtes Verhalten als Indikator für abweichendes Verhalten. In: Sack, F. / König, R. (Hrsg.), Kriminalsoziologie. Frankfurt a.M. 1968, S. 60-70

Shor, J.F. / Strodtbeck, F.L.: Group Process and Gang Delinquency. Chicago 1965

Simons, R.L. / Gray, P.A.: Perceived Blocked Opportunity as an Explanation of Delinquency Among Lower-Class Black Males: A Research Note. In. Journal of Research in Crime and Delinquency 26
(1990), S. 90 - 101

Singer, S.I. / Levine, M.: Power-Control Theory, Gender and Delinquency: A Partial Replication with Additional Evidence on the Effect of Peers. In: Criminology 26 (1988), S. 627-647

Smith, D. / Gartin, P. R.: Specifying Specific Deterrence: The Influence of Arrest on Future Criminal Activity. In: American Sociological Review 54 (1989), S. 94 - 105

Steffensmeier, D.J.: Crime and the Contemporary Women: An Analysis of Changing Levels of Female Property Crime 1960 - 1975. In: Social Forces 57 (1978), S. 566 -584

Steffensmeier, D.J. / Steffensmeier, H. R.: Trends in Female Delinquency: An Examination of Arrest, Juvenile Court, Self-Report, and Field Data. In: Criminology 18 (1980), S. 62 - 85

Sutherland, E.H.: Die Theorie der differentiellen Kontakte. In: Sack, F. / König, R. (Hrsg.): Kriminalsoziologie. Frankfurt a.M. 1968, S. 395-399

Sykes, G.M./Matza, D.: Techniken der Neutralisierung: Eine Theorie der Delinquenz. In: Sack,

F. / König, R. (Hrsg.): Kriminalsoziologie. Frankfurt a.M. 1968, S. 360-371
Tapp, J.L./Gunnar, M./ Keating, D.: Socialization: Three Ages, Three Rule Systems. In: Perlman,
D./Cosby, P.C. (Hrsg.): Social Psychology D. / Cosby, P.C. (Hrsg.): Social Psychology. New York 1983, S. $42-74$ Tapp, J.L./Kohlberg, L.: Developing Senses of Law and Legal Justice. In: Tapp, J.L./Levine,
F.J.(Hrsg.): Law, Justice and the Individual in Society. New York 1977

Thomae, H.: Formen der Auseinandersetzung mit Konflikt und Belastung im Jugendalter. In: Olbrich,

E. / Tcdt, E. (Hrsg.): Probleme des Jugendalters. Berlin etc. 1984, S.89- 110
Tittle, Ch. R. / Meier, R.F.: Specifying the SES / Delinquency Relationship. In: Criminolgy 28 (1990),
S. $271-299$

Tittle, Ch. R.: Social Class and Criminal Behavior: A Critique of the Theoretical Foundation. In: Social Forces 62 (1983), S. $334-358$

Tittle, Ch. / Villemez, W.J. / Smith, D.A.: The Myth of Social Class and Criminality: An Empirical Assessment of the Empirical Evidence. In: American Sociological Review 43 (1978), S. 643-656

Todt, E.: Selbstkonzept und Selbstkonzeptänderung als Mittler bei der Bewältigung von Anfordenungen in der Adoleszenz.In: Olbrich, E. / Todt, E. (Hrsg.): Probleme des Jugendalters. Neuere Sicht-
weisen. Berlin etc. 1984, S. 159 - 186

Toman, W.: Der psychoanalytische Ansatz zur Delinquenzerklärung und Therapie. In: Lösel, Fr.

(Hrsg.): Kriminalpsychologie. Weinheim-Basel 1983, S. $41-51$ 
Vanberg. V.: Verbrechen, Strafe und Abschreckung. Die Theorie der Generalprävention im Lichte der neueren sozialwissenschaftlichen Diskussion. Tübingen 1982

Wells. L.E. / Rankin, J.: Self-Concept as a Mediating Factor in Delinquency. In: Social Psychology 46 (1983), S. $11-22$

Wells, L.E. / Rankin. J.H.: Families and Delinquency: A Meta-Analysis of the Impact of Broken Homes. In: Social Problems 38 (1991), S. 71 - 93

Wells. L.E. / Rankin, J.H.: The Broken Home Model of Delinguency: Analytical Issues. In: Journal of Research in Crime and Delinquency 23 (1986), S. 68 - 93

Wells, L.E.: Self-Enhancement Through Delinquency: A Conditional Test of Self-Derogation Theory. In: Joumal of Research in Crime and Delinquency 26 (1989), S. 226-252

Wells. L.E.: Theories of Deviance and the Self-Concept. In: Social Psychology 41 (1978), S. 189 - 204

Whitehead, J.T. / Lab, St.P.: A Meta-Analysis of Juvenile Correctional Treatment. In: Journal of Research in Crime and Delinquency 26 (1989), S.276-295

Wilkinson, K.: The Broken Family and Juvenile Delinquency: Scientific Explanation or Ideology?. In: Social Problems 21 (1974), S. 726 - 739

Yablonski, L. : The Delinquent Gang as a Near Group. In: Rubington, E. / Weinberg, M.S. (Hrsg.): Deviance. The Interactionist Perspective. New York 1973, S. 245-255

Yablonski, L.: The Violent Gang. New York 1962 